\title{
An investigation into compassion fatigue and self-compassion in acute medical care hospital nurses: a mixed methods study
}

Katherine Valentine Upton

\begin{abstract}
Background: In the acute medical care hospital setting, nursing the sick and dying is both physically and emotionally demanding, making acute medical care hospital nurses more vulnerable to Compassion Fatigue (CF) or Secondary Traumatic Stress (STS). If not addressed in its earliest stages, CF can adversely change the caregiver's ability to provide compassionate care. It has been shown that Self-Compassion (SC) can be beneficial for the caregiver, with corresponding benefits for the individual needing care. However, the relationship of this attribute to CF in the acute medical care hospital nursing setting has not been intensively studied. This study explores the environmental and psychosocial factors affecting the prevalence and levels of CF in acute medical care hospital nursing staff and whether SC can be used as a coping strategy that enables nurses to mitigate the prevalence and levels of CF.

Methods: Using a mixed-methods study design, acute medical care hospital nurses were surveyed using a demographic/ work-related questionnaire, the Secondary Traumatic Stress Scale (used to measure CF), the Self-Compassion Scale, questions requiring a narrative written response and semi-structured informal interviews. One-way ANOVA was conducted to explore the impact of work-related and demographic characteristics on levels of SC and CF. Pearson correlation co-efficient ( $r$ value) was used to explore the relationship between CF and SC and lastly, multiple regression was used to discover whether a predictive relationship existed between SC and CF. The interviews were recorded, transcribed verbatim and subjected to thematic analysis.

Results: The results of this study have exposed the prevalence of CF in acute medical care nurses and it's damaging effects. And has also confirmed that SC can have a moderating effect on CF and an ability to be predictive of CF. Factors that influence the distress and vulnerability to CF were also identified, as well as the personal approaches used, whether successful or not, to enhance levels of SC and the 'barrier's to achieving it.
\end{abstract}

Conclusion: This unique study could lead to care environments encouraging the self-compassion and well-being of their acute medical care nursing staff and become places where threat cultures, weak leadership, an emphasis on task rather than process and a feeling of being undervalued, do not monopolise.

Keywords: Nursing, Nurse, Compassion fatigue, Secondary traumatic stress, Self-compassion, Compassion, Caring, Resilience, Wellbeing, Self-care

Correspondence: katevupton@aol.com

Visiting Lecturer at Aston Medical School, Aston University, Birmingham, UK

(c) The Author(s). 2018 Open Access This article is distributed under the terms of the Creative Commons Attribution 4.0 International License (http://creativecommons.org/licenses/by/4.0/), which permits unrestricted use, distribution, and reproduction in any medium, provided you give appropriate credit to the original author(s) and the source, provide a link to the Creative Commons license, and indicate if changes were made. The Creative Commons Public Domain Dedication waiver (http://creativecommons.org/publicdomain/zero/1.0/) applies to the data made available in this article, unless otherwise stated. 


\section{Background}

With recent examples of neglectful attitudes of health care staff towards vulnerable patients in the NHS [1-3], there has been a shift in focus to the importance of compassionate and respectful caregiving [4-7].

Compassionate care is more than being kind. It is an emotional connection that encompasses 'seeing the person within the patient' [8] and it has been shown to result in appreciative patients [9], better patient and caregiver experiences [10], a reduction in employee turnover [11] and improved patient clinical outcomes $[12,13]$. Shanafelt et al. [14] observed that clinicians' satisfaction with their relationships with patients could protect them against burnout, professional stress, substance abuse and even suicide attempts.

Advances in medical science have enabled patients with multiple comorbidities to be provided with more active treatment. However, this has consequently required that patients in hospital care, on average, need more intensive care management [15]. This is of significance in the acute medical care hospital ward setting, where nurses care for the sick and dying in a work environment that faces sustained increasing pressure on health expenditure from an ageing population, the introduction of new technology (which is often complex) and rising public expectations [16].

Working in such an environment is both physically and emotionally demanding and, within which, compassionate nursing care is an expected professional and moral obligation of a nurse's day-to-day practice. It is an expectation of the patient and the public as a whole, and openly stated as central to both political and professional drivers $[6,7]$.

Unfortunately concerns about lack of compassionate patient care $[3,17]$ have exposed examples of the neglect of vulnerable people and have been exacerbated by media reports condemning nurses [18].

Although health care professionals provide care to patients "in the context of organisations, not in isolation" [19] (pp. 1), nurses have been scapegoated in those organisational cultures that emphasise a performance culture of individual blame [20]. Indeed, conflict between policy and current evidence on compassionate care and cultural change is all too evident in a Governmental review [21], which was commissioned to advise NHS England on how to protect patients from harm and, it's findings pointed to a complete system failure, rather than finding fault at the individual level [21].

\section{The elements of compassion and compassionate care}

The concepts of 'compassion' and 'compassionate nursing' are complex and difficult to define [21-23]. Lewin [24] defines 'compassion' as a basic central value that is combined with the complication of emotions. It is seen as being dependent on an individual's capacity to develop a compassionate mentality and orientation [25], involving such attributes as attention, awareness, motivation, wisdom, commitment and courage [26].

Compassion for friends and families is distinctive in nature [27], as it involves helping and supporting those with whom an individual has a close emotional attachment [28]. However, the compassion one finds within health care and nursing is more complicated and multi-faceted [28], involving individuals who, invariably, have no historical connection $[29,30]$.

Compassion has been called "Nursing's most precious asset" [31] (pg. 48), involving entering and submerging into a patient's experience and feelings [32] and engaging in activities to relieve their anguish and discomfort [33]. Indeed, it is the presence of "small acts of kindness" [34] (pg. 6), that take on meaning and mark a patient's and his/her family's vivid and continuing memories of the overall experience of the health care received.

The negative impact on a nurse caring for those experiencing traumatic stress and suffering, can be the development of 'Compassion Fatigue', a phenomenon with signs and symptoms similar to those of Post-Traumatic Stress Disorder [35].

A tool to measure the presence of Compassion Fatigue has been developed, the Secondary Traumatic Stress Scale (STSS) [36].

\section{Concept of compassion fatigue}

The effect of Compassion Fatigue (CF) is multifaceted, including physical, emotional, social, spiritual and intellectual effects. Symptoms of CF include; boredom, cynicism, anxiety, discouragement, intrusive thoughts, irritability, avoidance, numbness, persistent arousal, sleep disturbances, depression, intolerance, detachment, apathy and, not least, a loss of compassion [37-41]. Physical symptoms can include increased blood pressure, weight gain, fatigue, stiff neck, immune dysfunction and, an increase in gastrointestinal problems, cardiovascular disease and diabetes [42].

It has been shown that CF not only takes its toll on health care professionals on a personal level, but also on the workplace itself, causing more sick days, a higher turnover rate, decreased productivity [43], changes in job performance, patient dissatisfaction, poor professional judgment and an increase in mistakes [44-47].

In order to self-manage the emotional and physical symptoms of CF, there is concern that individuals may adopt ineffective coping strategies, such as avoidance, withdrawal and emotional numbing, which can then have a negative impact on the quality of care a patient receives [38].

Up to $85 \%$ of healthcare workers display CF [39], with direct consequences for patient care, termed the 'cost of 
caring' $[48,49]$. As sustained responders, nurses are particularly vulnerable to CF [50], which, if not addressed in its earliest stages, can permanently change the caregiver's ability to provide compassionate care [37]. However, in most British hospitals, nurses have little or no formal support to counter the potentially negative emotional consequences of their work $[42,47,51]$.

\section{Concept of self-compassion}

Self-Compassion (SC) was defined by Neff $[52,53]$ as a construct that plays a significant part in how people deal with life's difficulties. Based on Buddhist philosophy, SC, in its simplest terms, is compassion directed inward [54], with oneself being the object of care and concern [52]. SC consists of three main elements; 'Self-Kindness' which refers to self-soothing and self-caring, a sense of 'Common Humanity', which involves recognising that no one is perfect and that we all make mistakes, and 'Mindfulness', being aware of being in the present moment. Instead of reacting to an unpleasant situation with self-criticism and harshness, a person with SC puts suffering into perspective, acknowledging and accepting that $\mathrm{s} /$ he is not unique in suffering and having inadequacies. Subsequently s/he shows kindness and compassion towards his/herself [55].

SC has been shown to contribute to well-being, alleviating depression and other psychological conditions $[52,55-58]$. It is associated with psychological strengths, such as emotional intelligence and resilience, optimism, wisdom, motivation, personal initiative and curiosity and exploration [56, 59-61]. SC has also been shown to improve how one relates to others [62] and encourages more empathic concern and altruism [63]. In nursing situations, such as care of the dying, one could predict that, with a high degree of SC, a nurse will be less likely to avoid and disconnect from the suffering of the patient and family.

Of particular importance is that an attribute of $\mathrm{SC}$ is 'self-mastery', the willingness to learn from failure and a willingness to try again without self-recrimination. Consequently, SC may be vital for protecting an individual from Compassion Fatigue and thereby promoting compassionate patient care [64].

A tool to measure the level of Self-Compassion has been developed, the Self-Compassion Scale (SCS) [52]. However, the relationship between Self Compassion and Compassion Fatigue in the delivery of care by nurses has not been intensively studied.

\section{Purpose of the study}

The aim of this study is to address gaps in previous CF and SC research by examining their prevalence and levels in acute medical care hospital nurses. Acute medical care hospital nurses were chosen as opposed to any other nursing specialty, as it was evident on conducting a systematic literature search, that no previous research had been conducted on $\mathrm{CF}$ and SC in relation to nurses who work within the acute medical care hospital ward setting, in the NHS. Nurses working in this care environment often provide care for patients who have multiple and complex needs, often seriously ill or dying, whilst providing support for the patients' families and loved ones. The acute care medical environment is often fast-paced and emotionally, intellectually and physically challenging [65], with an ageing population that is predicted to experience more chronic disease and physical incapacity [66].

Acute medical care nurses need to be alerted to the potential of CF and its effects, on their feelings and behaviour. Although researchers have begun to study the possible moderating effects of $\mathrm{SC}$ on resilience and coping [67], only one study has focused its attention on the prevalence of SC in registered nurses (Heffernan, et al., 2010). Hitherto, studies investigating CF in healthcare have focused on specialist nurses, particularly oncology [41, 47, 68] and emergency nurses [39].

This study also explores whether factors, such as demographic and work-related characteristics, contribute to the development of CF and SC in acute medical care hospital nurses. It investigates the personal approaches the nurses use to achieve SC and whether there are barriers to accomplishing it. The study also examines whether the level of SC can predict CF in acute medical care nurses. If statistically demonstrated, SC level could become a screening tool to identify those most vulnerable to developing CF.

Furthermore, the study seeks to examine how SC can act as a coping strategy, enabling acute medical care hospital nurses to cope better with CF. Such information could provide healthcare organisations with an efficient approach to targeting interventions that can increase SC in acute care medical hospital nurses, thereby improving the nurse-patient relationship [60].

\section{Methods}

\section{Research questions}

An Integrative Literature Review (ILR) was conducted to evaluate the research literature related to Compassion Fatigue (CF) and Self-Compassion (SC), and identify the best research methods to gain an understanding of the prevalence and levels of $\mathrm{CF}$ and $\mathrm{SC}$ in acute medical care hospital ward nurses.

After considering the ILR findings, the following research questions were developed:

\footnotetext{
$\triangleright$ What are the environmental and psychosocial factors affecting the prevalence and levels of Compassion Fatigue in acute medical care hospital nursing staff?
} 


\section{$>$ Is Self-Compassion a coping strategy that enables acute medical care hospital nurses to mitigate the prevalence and levels of Compassion Fatigue?}

\section{Design}

A mixed methods research design was chosen, with the findings presented both statistically and thematically. Triangulation of the quantitative and qualitative data was carried out to increase the enquiry's scope and comprehensiveness [69] to provide adequate trustworthiness, so enabling the limitations of one type of data to be balanced by the strengths of the other [70]. Figure 1 diagrammatically displays the research design of this study, including the total number of participants involved at each stage of the process.

\section{Setting}

The study was conducted at a large, urban, University general hospital in the United Kingdom. It serves as a Level I Trauma Centre and as a teaching hospital with over 1200 inpatient beds.

\section{Participants}

The study sample was recruited from a target population of registered nurses (RNs) working in an acute medical care hospital setting. In total, five acute medical care hospital wards were identified, providing approximately 180 potential study participants. These chosen wards did not include acute medical care wards that either specialised in cancer care, Accident and Emergency or Critical Care. Inclusion criteria for participation were used (Table 1).

\section{Sample recruitment}

After approval of the hospitals' ethics committee, the nurse ward managers of the selected wards were directly contacted by the researcher and the study aims, recruitment methods and the importance of participation, were explained.

With consent, flyers were placed around the wards and when appropriate, engagement with potential participants took place. Information packs and questionnaire packs (Table 2) were either handed out directly to the potential participants or placed in full view in the nurse staff rooms.

In line with ethical requirements, each questionnaire pack was proceeded by an information sheet describing the study aims and assuring the RGNs that the information they provided would be kept confidential and anonymous, and that their participation was voluntary. They were informed that not all participants would be approached for the interview stage of the study, even if consent were given.
A sealed box was placed on each ward for the completed questionnaires and 'expression of interest' form to be placed.

\section{Data collection}

Quantitative data was collected between 29.09.15 to 17.11.15 using a questionnaire pack which included demographic and work-related questions, the Secondary Traumatic Stress Scale (STSS) [36], to measure the incidence and levels of Compassion Fatigue (CF), and the Self-Compassion Scale (SCS) [52], to measure the incidence and levels of Self-Compassion (SC). To optimise the response rate and to address potential sample recruitment barriers and challenges [71], a number of strategies were implemented, including regular visits to the wards and emails to the nurse ward managers. At the end of the 4-week data collection period, the 5 sealed boxes were collected, their seals broken and, the completed questionnaires and 'expression of interest' to be interviewed forms counted.

The STSS and SCS scores for each of the participants were calculated and interpreted using the score categories developed by the creators of the two tools [36, 52]. The intention was to select from those who had completed the 'expression of interest' form, those participants who typified a particular score in both the STSS and SCS. The rationale for adopting this approach was to explore emerging themes characterising the narratives of low, moderate and high levels of CF (STSS) and SC (SCS). However, with a poor response rate, this strategy was not possible and all potential interviewees were contacted and asked to complete a Doodle Poll to organise their interview date/time.

Qualitative data was collected using written questions requiring a narrative response and one-to-one semi-structured interviews to provide a more intensive and comprehensive picture to illustrate the findings obtained from the quantitative data. The interviews took place in quiet and private locations and were audio-recorded using two devices to protect against the possibility of one of the devices failing during the interview. Prior to the interview participants were assured anonymity and confidentiality, the purpose and process of the interview were explained, and an interview consent form completed.

\section{Instruments}

\section{Quantitative strand: questionnaire}

The questionnaire was divided into four sections. Demographic and work-related variables were selected for the first section and the final two questions were related to whether the participant had been previously aware of the terms CF and SC. The rationale for this was based on evidence from a study [68], which found that more than $50 \%$ of oncology nurses had not heard of the term Compassion Fatigue. 


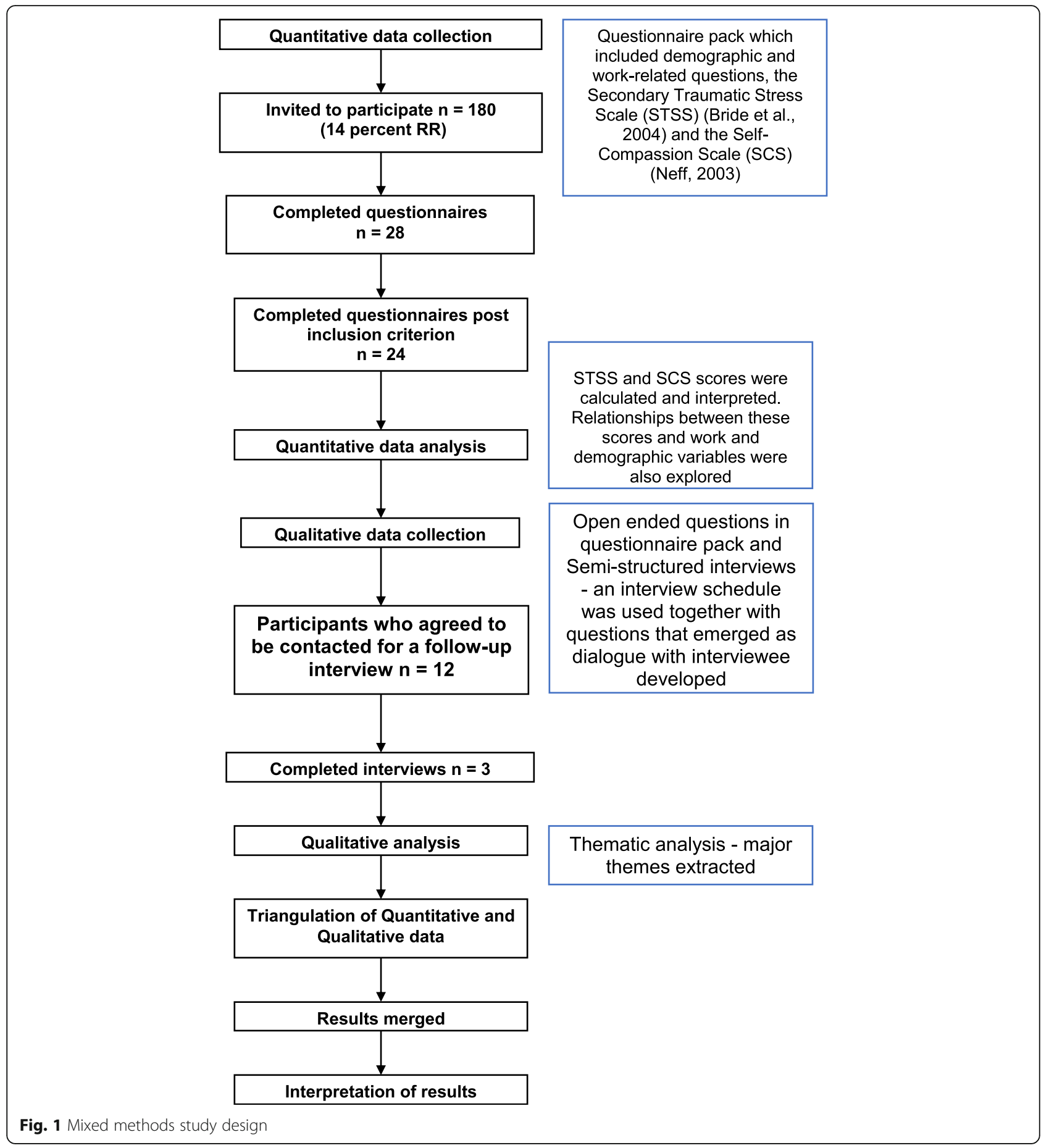

The second section included the Self-Compassion Scale (SCS) [52]. The scale includes six subscales of 'Selfkindness' versus 'Self-Judgment', 'Common Humanity' versus 'Isolation' and 'Mindfulness' versus 'Over-identification'. This enables individuals to be scored on each of the subscales, in conjunction with the main objective of the scale, which is to measure SC as a single principal construct (Neff, 2003a). The SCS is a 26-item tool that identifies 3 components of SC. Participants were instructed to rate how well each statement (item) described them using a 5-point Likert scale, ranging from 1 ('almost never') to 5 ('almost always'). A Cronbach's alpha coefficient of 0.92, has been identified by Neff (2003b) along with the following for the subscales; isolation (0.79); self-judgment (0.77); over identification (0.81); self-kindness (0.78); common humanity (0.80); and mindfulness (0.75) [52]. 
Table 1 Inclusion criteria for participation

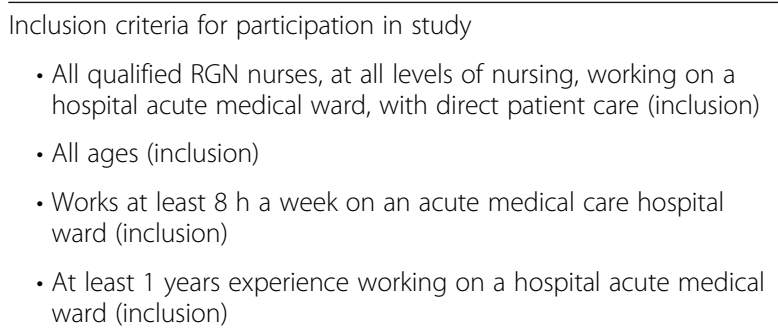

The third section of the questionnaire, the Secondary Traumatic Stress Scale (STSS), is the only instrument that has been designed to assess Secondary Traumatic Stress (STS) [36]. The symptoms of STS are virtually identical to those of Post-Traumatic Stress Disorder (PTSD) and include symptoms of 'Avoidance', 'Intrusion' and 'Arousal' [35]. Compassion Fatigue [35] is the term now commonly used to describe Secondary Traumatic Stress Disorder (STSD), which results from caregivers indirectly experiencing the trauma of their seriously ill and/or dying patients and families [41]. The 17-item instrument has been designed to measure the subscales of 'Intrusion', 'Avoidance', and 'Arousal' symptoms. In this study, participants rated how frequently they experienced each symptom in the past 7 days, ranging from 'never' to 'very often'. Each of the STSS 17 items and 3 subscales corresponds with criteria $\mathrm{B}, \mathrm{C}$ and $\mathrm{D}$ in the Diagnostic and Statistical Manual of Mental Disorders [72], used in the diagnosis of Post-Traumatic Stress Disorder (PTSD). The STSS has demonstrated construct validity through convergent, discriminant and factor analyses $[36,73]$ and high levels of internal consistency reliability $[38,74,75]$.

\section{Qualitative strand: written narrative responses and semi structured interviews}

The final section of the questionnaire asked the participants to share their thoughts on the phenomena under investigation by giving a narrative response to open-ended questions.

In the semi-structured interviews, open-ended questions were used, together with questions that emerged as

Table 2 Content of the information and questionnaire packs

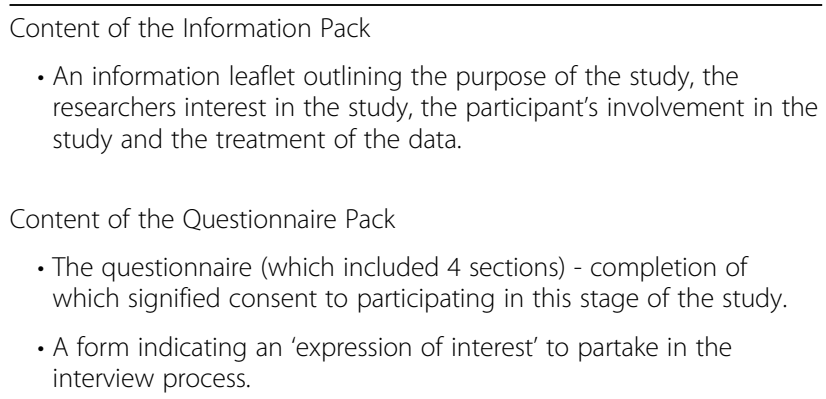

- An information leaflet outlining the purpose of the study, the researchers interest in the study, the participant's involvement in the study and the treatment of the data.

Content of the Questionnaire Pack

- The questionnaire (which included 4 sections) - completion of which signified consent to participating in this stage of the study.

- A form indicating an 'expression of interest' to partake in the interview process.

the dialogue with the interviewee developed $[76,77]$ in order to explore the nuances and subtleties of the phenomena under investigation that the quantitative tools were unlikely to reveal. Field notes were taken of any non-verbal cues expressed by the interviewee that could be used when transcribing and interpreting the data. All interviewees were given the opportunity to provide as many explanations and examples as they wanted [78]. A reflexive diary was also used at the analysis stage to review whether the researcher's own history and feelings had possibly influenced the interview outcome [79].

\section{Data analysis \\ Quantitative strand}

Total Secondary Traumatic Stress Scale (STSS) scores for each individual participant were calculated by summing the response value for each item (no reverse scoring is used in the STSS). The total scores for each of the 3 subscales ('Avoidance,' 'Intrusion' and 'Arousal') were also calculated. Categories developed by Bride et al. (2004), were used for the interpretation of the total calculated STSS scores.

Calculation of the total Self-Compassion Scale (SCS) scores involved the reverse scoring of the negative subscale items of 'Self-judging', 'Isolation' and 'Over-identification' (i.e. $1=5,2=4,3=3,4=2,5=1$ ) and a grand mean of all six subscales was calculated (Neff, 2003a). The mean of each of the 6 subscales were also calculated. Instructions on interpreting SC scores developed by Neff [52] were used. Higher SCS total scores signified greater SC [52].

IBM SPSS Statistics Version 21.0 (SPSS, Chicago, Illinois) was used to analyse the data. Descriptive and frequency statistics were used to describe the study population demographic and work-related characteristics. One-way ANOVA explored the impact of the demographics on levels of SC and STS. Using Pearson correlation co-efficient ( $r$ value) and Cohen's [80] guidelines for the interpretation of the results, the relationship between the mean total SCS scores and the mean total STS scores was tested. Also, the association between participant demographic and work-related characteristics and the mean total STS and SCS and their subgroup mean scores. Finally, multiple regression was used to investigate whether a predictive relationship existed between Self-Compassion and Compassion Fatigue (STS).

\section{Qualitative strand}

Having transcribed the interviews verbatim, in order to gain greater insight into the experiences and views of the study participants, Thematic Analysis (TA) was used as a step-by-step framework to identify, organise, analyse and report patterns ('themes') within the data collected [81]. Proceeding through the six phases of TA [81], an inductive approach to data coding and analysis was used. 
Through a 'bottom-up' approach, patterns and consistencies were discovered, which guided the growth of hypotheses, which were subsequently explored and developed into general conclusions and theories. To improve study rigor, the researcher obtained supervisory support during the thematic analysis process for peer review purposes.

\section{Results}

Quantitative strand

\section{Sample characteristics}

Of the 200 questionnaires distributed, 28 were completed, representing a response rate of $14 \%$. However, 4 questionnaires were rejected as the respondents did not meet the inclusion criterion of qualified RGN nurse. The small sample size limits the generalisability of the quantitative findings. However, analysis of the data, enabled patterns to be identified and differences reported.

\section{Secondary traumatic stress scale (STSS) Total STSS and STSS subscale scores}

The results revealed that nearly $46 \%$ of the respondents had a mean total STSS score that fell within the range of 41 to 57 , indicating that they had moderate to very severe levels of STS [82].

\section{Diagnostic criteria and individual symptoms}

Respondents' scores were analysed to determine the presence of the diagnostic criteria for PTSD. As defined in the DSM-IV-TR [72], an individual must display at least one criterion B symptom ('Intrusion'), three criterion C symptoms ('Avoidance') and two criterion D symptoms ('Arousal'), at a level of 3 ('occasionally') or higher, to meet the diagnostic criteria for PTSD. $37.5 \%(n=9)$ met all 3 criteria and therefore were judged to have a diagnosis of PTSD. The most frequently reported individual symptoms, included, intrusive thoughts (Intrusion), discouraged about the future (Avoidance) and, difficulty sleeping, irritability and hypervigilance (Arousal).

\section{Demographic/work-related characteristics and mean total STSS scores}

The relationship between work and demographic variables and total STSS scores was tested using Pearson correlation co-efficient ( $r$ value). Using Cohen's [80] guidelines for interpretation, a moderate, positive correlation (relationship) was found between the mean total STSS scores and age $(r=0.420)$, with years-experience working on an acute medical care hospital ward $(r=0.313)$ and with hours worked per week $(r=0.436)$. The results also indicated that STS levels rise sharply from mid-30s, reaching a peak in the early forties, which is then sustained through to the mid-fifties (Fig. 2). Those new to the acute care medical hospital setting $(n=3 / 12.5 \%)$ reported very high, moderate levels of STS and an average total STSS score of 45.13 was experienced by those who had between 12 and 22 years of acute medical care ward experience (Fig. 3). Higher levels of STS related to short part-time work and full-time working patterns (Fig. 4).

The study also showed that those who reported themselves as 'RGNs' (25\%), in relation to 'Professional Qualification', had high levels of STS, followed by those who reported to having a Nursing Degree, who had a moderate level (41.0).

\section{Demographic/work-related characteristics and mean total STSS subscales scores}

A significant difference at the $p<0.05$ level between the mean Criterion B Intrusion subscale total and professional qualification was found using the one-way ANOVA test $(p=0.012)$. Those describing their qualification as a 'Nursing Degree' had the highest mean Intrusion subgroup score (14) (Fig. 5).

Those aged 41-45 years (Fig. 6) and those who had the longest work-experience (22 years) on an acute medical care hospital ward (Fig. 7), showed the highest levels of Avoidance (Criterion C). In terms of age, Arousal levels (Criterion D) steadily increased from a low level (6.0) at 31 years of age, to those aged 51 to 55 years (17.0) (Fig. 8).

\section{Self-compassion scale (SCS) \\ Total SC and SC subscale scores}

The mean SCS score was 2.87, indicating a lower end of moderate level of SC. $71 \%$ of respondents had a SC score of 2.5 and above, indicating moderate to high levels of SC in the majority of subjects. Table 3 details the scores for each of the SCS subscales and the mean value for each.

Drilling down into specific SCS questionnaire items, 'Keeping emotions in balance' (item 9), part of the Mindfulness subscale, had the highest mean score (3.42) within the positive subgroups. This indicated a high moderate level of SC being experienced frequently by $87.5 \%$ of respondents. However, 54\% had 'almost never' or 'rarely' 'tried to approach their feelings with curiosity and openness when they were feeling down' (item 22), which was reflected in the item's mean total score (2.42), signifying low SC.

Within the 'Self-kindness' positive subscale, low levels of $\mathrm{SC}$ were found for 'I try to be loving towards myself when feeling emotional pain' (item 5) and 'I'm kind to myself when I'm experiencing suffering' (item 19). And within the 'Common Humanity' subscale, between 50 and $75 \%$ of respondents reported high scores in all 4 items.

For the three negative subscales, $75 \%$ of respondents, demonstrated low self-compassionate behaviour towards oneself (item 16). Between 62 and $75 \%$ of respondents 


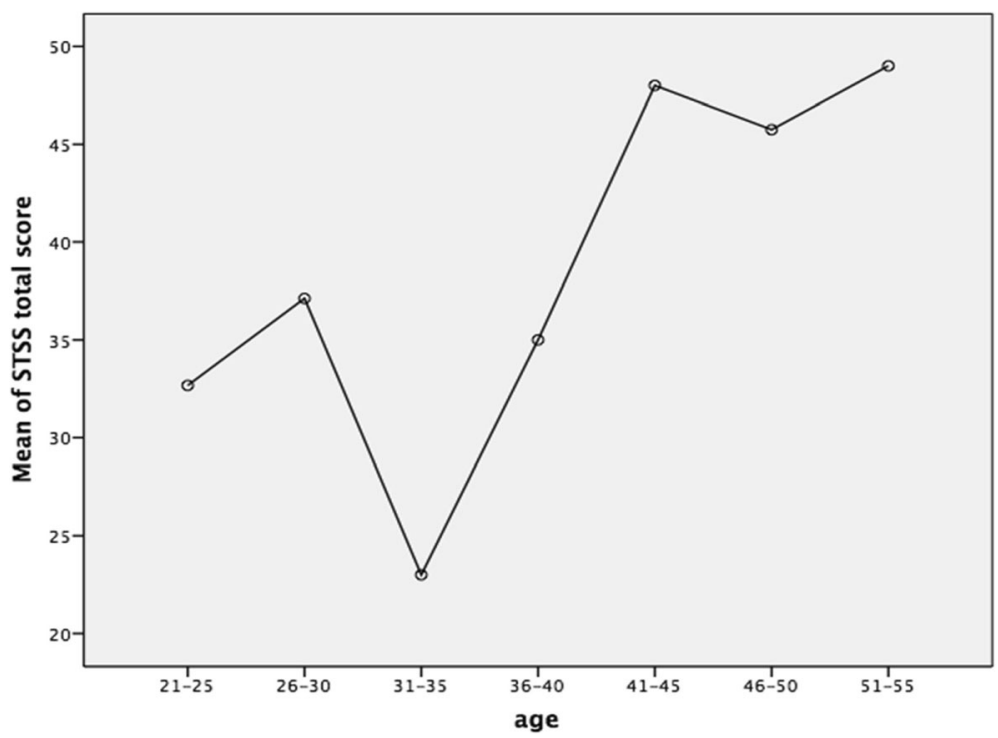

Fig. 2 Mean total STSS scores across age groups

frequently reported 'Over-identification', and between 46\% (item 4) and 75\% (item 18) reported 'Isolation'.

\section{Demographic/work-related characteristics and mean total}

\section{SCS scores}

Of particular significance, those aged $31-35$ years $(4.2 \%)$ (3.57) (Fig. 9) and those who had $3(n=1)$ and 10 years $(n=1)$ work-experience (3.64 and 3.57 respectively) (Fig. 10), had high levels of SC. In contrast, those aged
51-55 years $(4.2 \%)(2.21)$ and those with $6.5(n=1)$ and $7(n=1)$ years-experience (1.80 and 1.83 respectively) had considerably lower levels of SC.

Respondents who were married had the highest total mean SC score (3.10).

In contrast, those reported to be divorced (4.2\%) had the lowest total mean SC score of 1.83, indicative of considerably low levels of SC. Those who reported they were religious had a lower level of SC (2.65), than those who

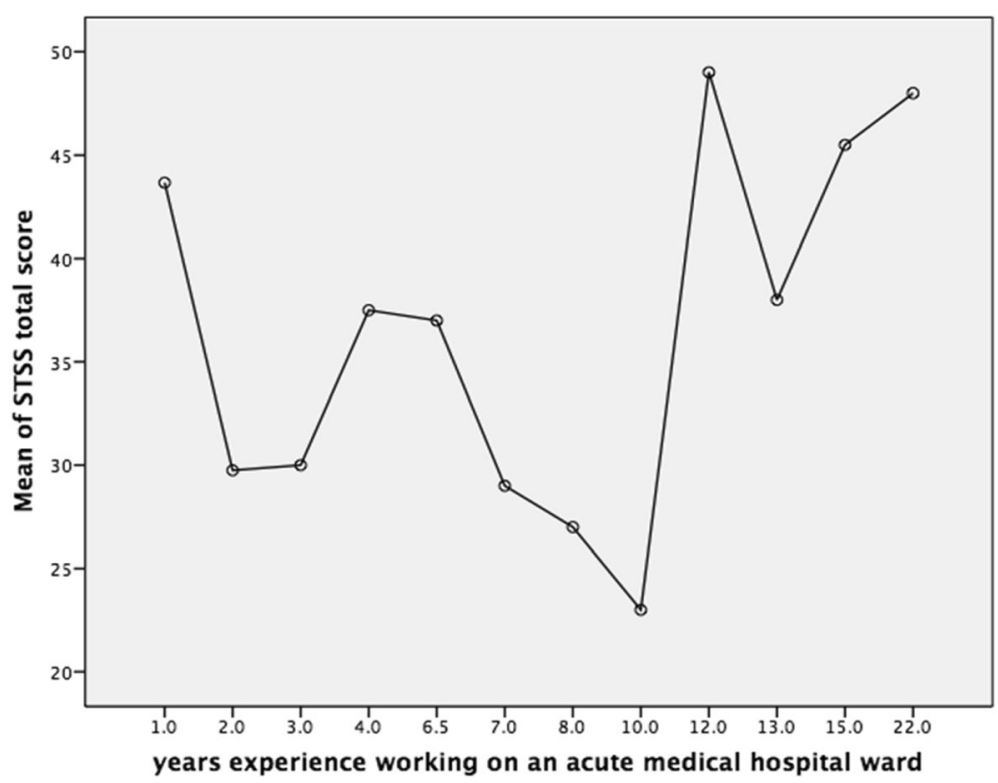

Fig. 3 Mean total STSS scores against years worked on acute medical ward 


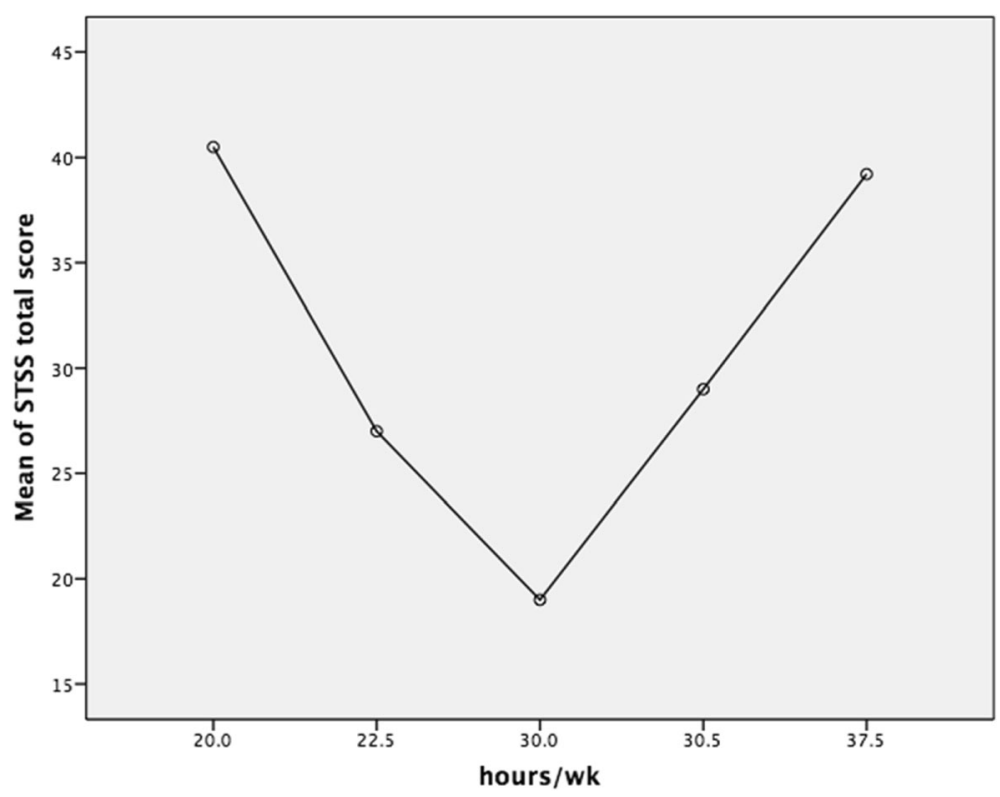

Fig. 4 Mean total STSS scores against hours work/week on acute medical hospital ward

reported not being religious (2.91). Those respondents, who described having a nursing degree had the lowest total mean SC score (2.75) of all the professional groups. In relation to the participant's 'Shift Pattern', 13 (54\%) who worked Full-time/day had moderate levels of SC (3.03).

\section{Demographic/work-related characteristics and mean total} SCS subscales scores

Those aged 31-35 (4.2\%) had the highest mean 'Mindfulness' subscale score (3.5) and the lowest mean scores in the three negative subgroups. In contrast, those aged 51-55 years (4.2\%) had the highest mean scores for the SC subscales of 'Isolation' (3.75) and 'Over-identification' (3.5), indicating less $\mathrm{SC}$ in this age group than any other. To illustrate, Fig. 11 demonstrates that the 'Common Humanity' score increased with age until it dropped steeply to those aged over 50 years. Those who were married were the most 'Self-Judging' (2.72) and those who were divorced had subgroup mean total scores indicating low levels of SC. Religious participants (16.67\%) had the

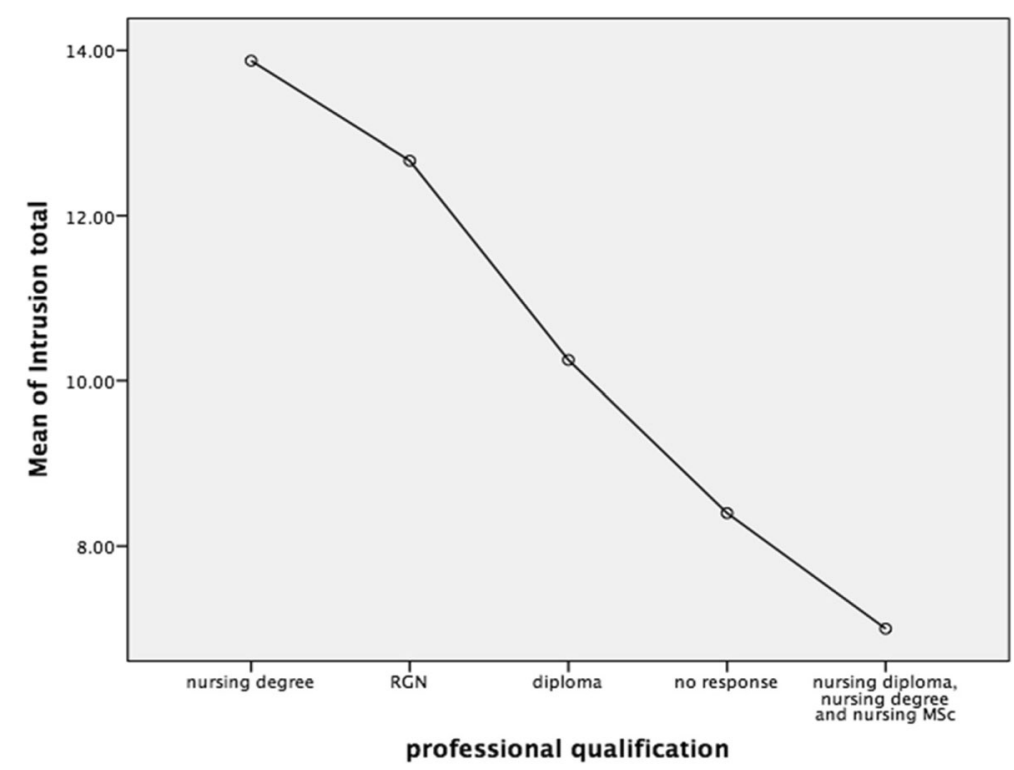

Fig. 5 Criterion B 'Intrusion' subscale mean totals against professional qualification 


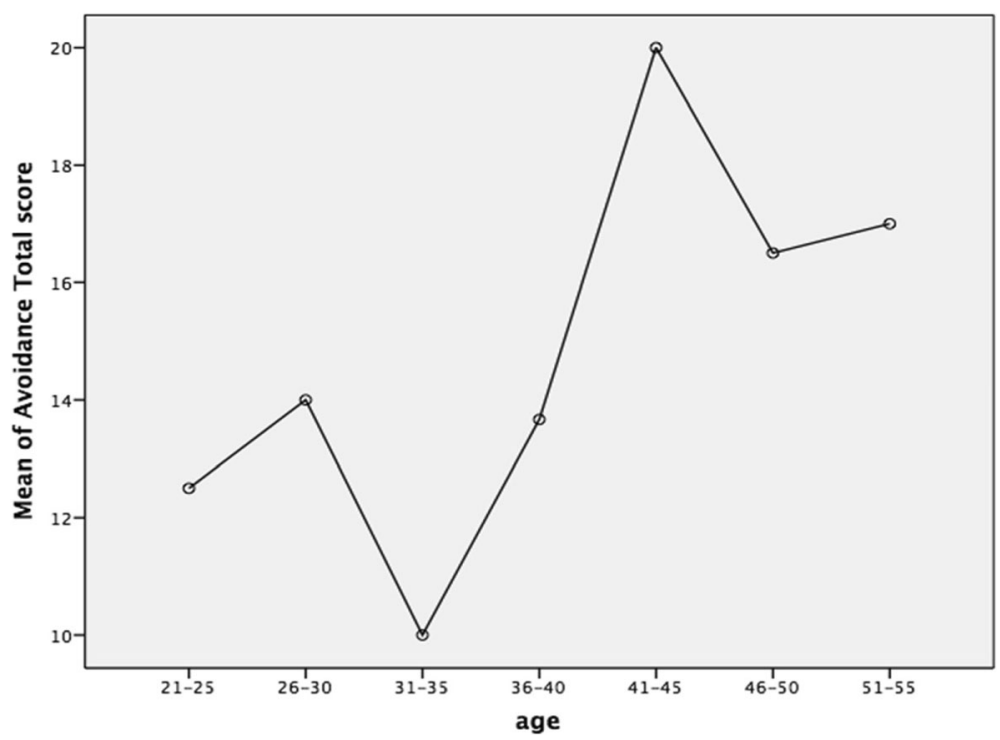

Fig. 6 Criterion C avoidance subscale mean totals against age

highest total mean scores in the positive SCS subscales, but also scored the highest in all the negative subscales.

\section{Prior knowledge of the terms compassion fatigue and self-compassion}

$58.3 \%$ of the respondents had not previously heard of the term Compassion Fatigue. They also had the highest mean total STSS score (39.79), indicating moderate levels of Compassion Fatigue. In relation to Self-Compassion, $70.8 \%$ of the respondents had not previously known of the term. Both those who had previous knowledge of the term Self-Compassion (2.91) and those who had no prior knowledge (2.84), had moderate levels of Self-Compassion.

\section{The relationship between STSS and SCS scores}

Using One-Way ANOVA, a graph to compare the mean total scores on STSS and SCS was created (Fig. 12).

The relationship between Compassion Fatigue and Self-Compassion was investigated using Pearson productmoment correlation co-efficient ( $r$ value). Using Cohen's

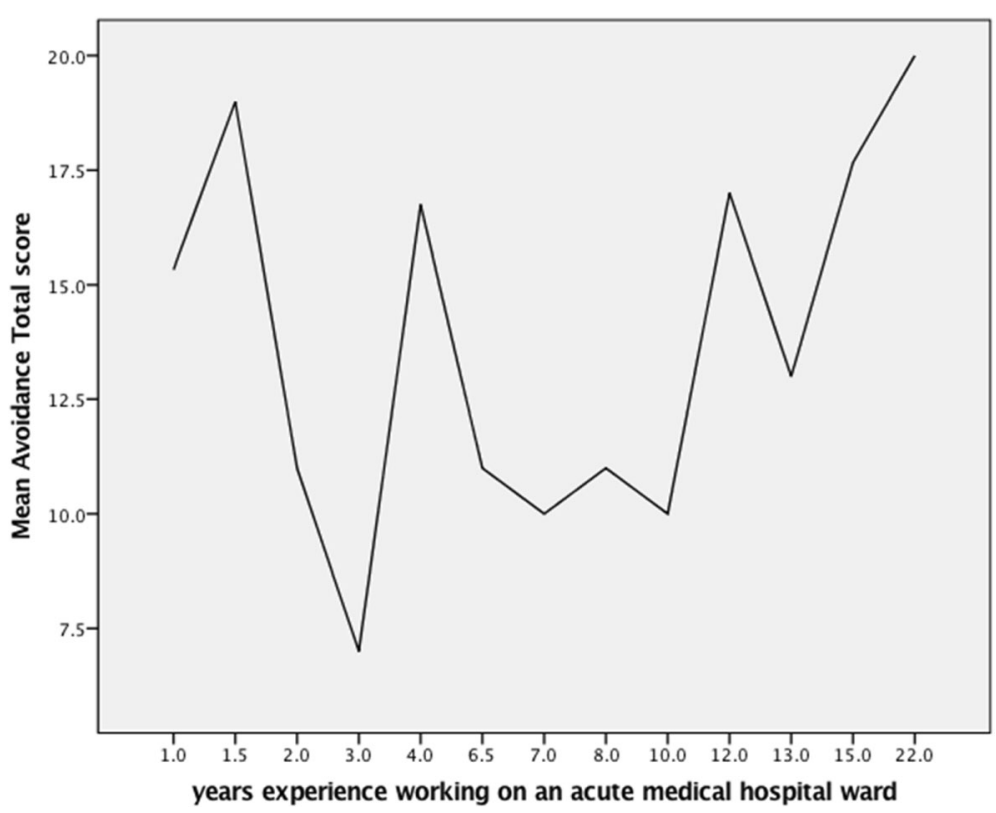

Fig. 7 Criterion C avoidance subscale mean totals against experience working on an acute medical ward 


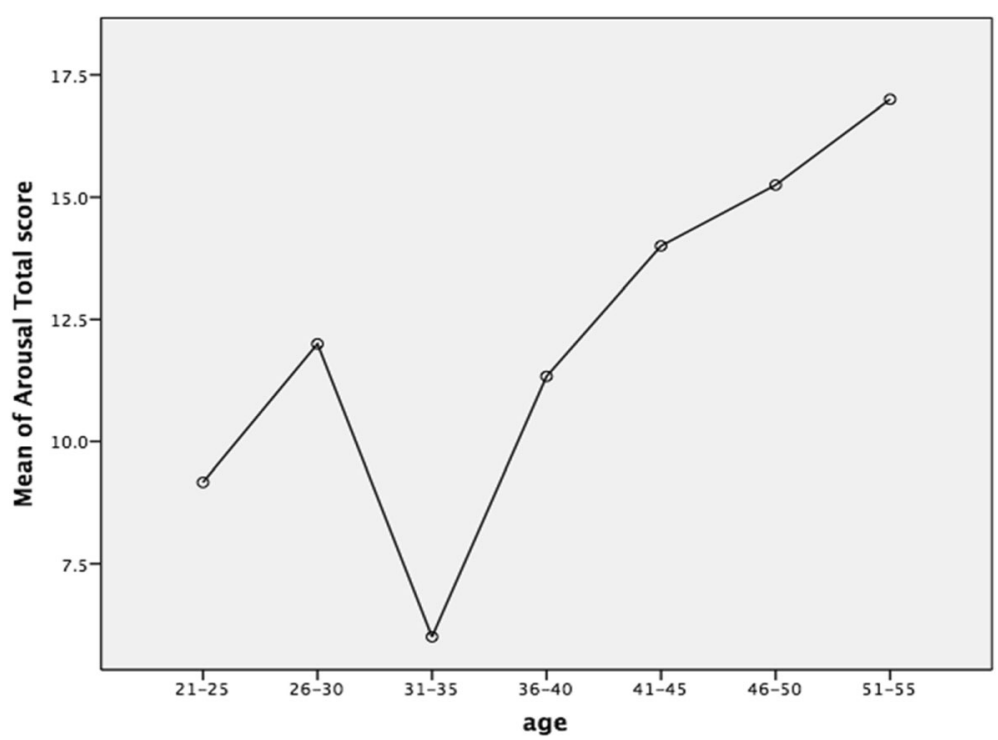

Fig. 8 Criterion D arousal subscale mean totals against age

(1988) guidelines for the interpretation of the results, a moderate, negative correlation between the two variables was found $(r=-0.445, n=24, p<0.029)$, with high levels of Self-Compassion associated with lower levels of Compassion Fatigue.

In order to explore the predictive power of Self-Compassion (independent variable) in relation to Compassion Fatigue (dependent variable), standard multiple regression was used. The result of this test showed a Sig. value of 0.029 , a value less than 0.05 , indicating that Self-Compassion (independent value) is making a significant unique contribution to the prediction of Compassion Fatigue (dependent variable) (Fig. 13).

\section{Qualitative strand}

Of the 24 questionnaire respondents, 12 agreed to be contacted for a follow-up interview. An extended recruitment process resulted in the final interview response rate of $25 \%$ $(n=3)$. Despite the limited sample size, the three interviews gathered rich and thick data [83] that was meaningful enough to answer the aims and objectives of the study.
Within the transcribed interview data, both semantic and latent quotes were coded if significant and meaningful. These were grouped into six themes that were pertinent to the two research questions.

\section{Research question 1: environmental and psychosocial} factors affecting the prevalence and levels of CF in acute medical care hospital nurses

Three dominant themes emerged from the coded data related to Compassion Fatigue (CF). These were:

- Recognition of the existence of Compassion Fatigue.

- Presenting features of Compassion Fatigue.

- Factors contributing to the development of Compassion Fatigue.

\section{Theme 1: the existence of compassion fatigue}

All three participants were unaware of the term 'Compassion Fatigue' (CF) before the study. However, when they read the promotional study material and saw the study flyer, they recognised and identified with the

Table 3 SCS subscale maximum and minimum scores and means

\begin{tabular}{lllll}
\hline & Minimum SCS score & Maximum SCS score & Mean SCS score & Std. deviation \\
\hline Self-kindness Total Score & 1.00 & 3.60 & 2.47 & 0.67158 \\
Common Humanity Total Score & 2.00 & 5.00 & 2.95 & 0.79393 \\
Mindfulness Total Score & 1.75 & 4.75 & 2.95 & 0.68754 \\
Self-judgment Total Score & 1.40 & 4.80 & 3.16 & 0.77117 \\
Isolation Total Score & 1.25 & 4.75 & 3.00 & 0.84055 \\
Over-identification Total Score & 1.25 & 4.75 & 2.98 & 0.86576 \\
\hline
\end{tabular}




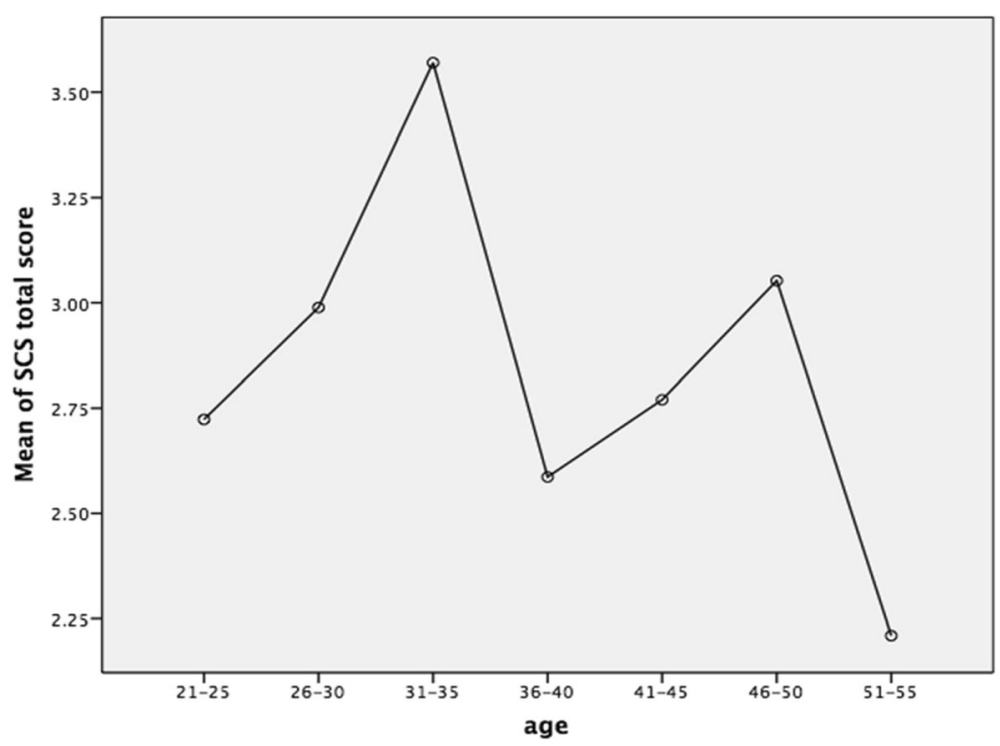

Fig. 9 Mean total SCS scores against age

term and its presenting features, making personal interpretations.

\section{Theme 2: presenting features of compassion fatigue}

When the participants were asked how they would describe their working lives, thematic analysis revealed four significant, but inter-related themes. These were:

i. Emotional Effects

ii. Cognitive Effects iii. Behavioural Effects

iv. Physical Effects

\section{Emotional effects}

The participants described feeling 'emotionally tired' with their work on the acute medical wards and a sense of 'emotional numbness'.

Overwhelming...with so many emotions flying around...[I] couldn't keep on top of them.

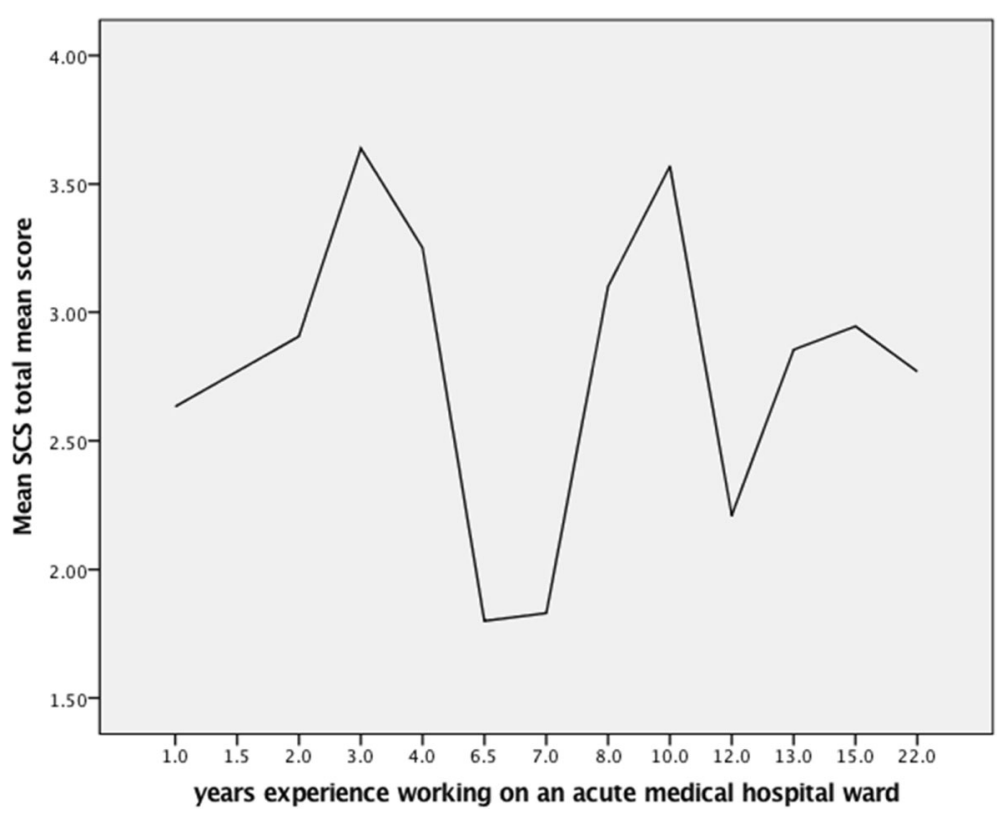

Fig. 10 Mean total SCS scores against years-experience working on an acute medical ward 


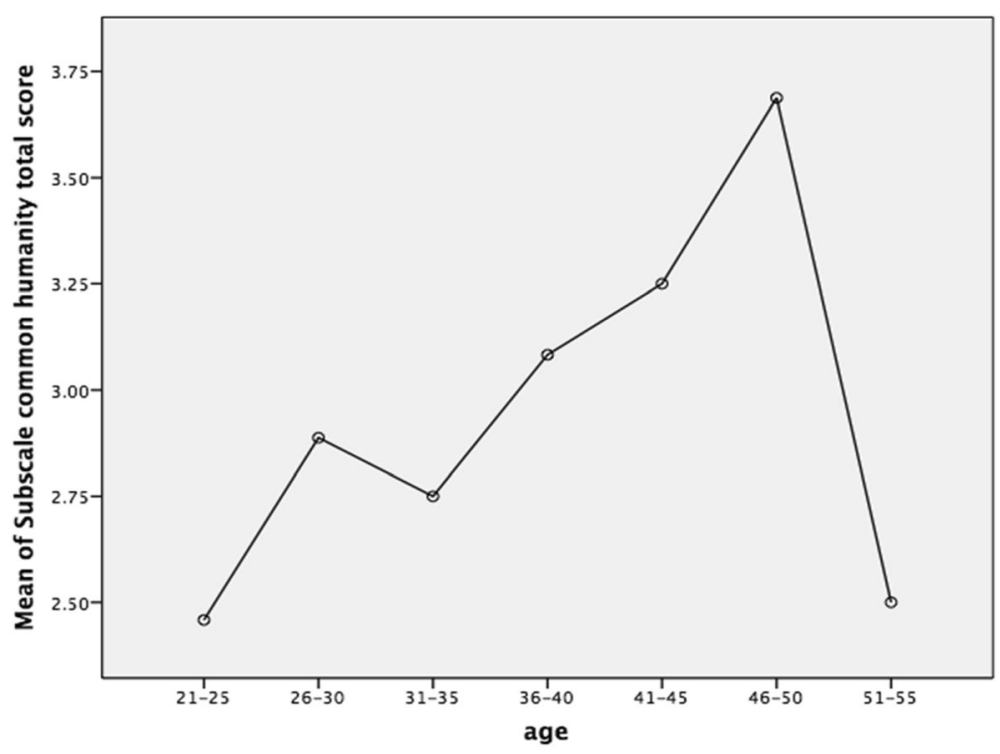

Fig. 11 SCS 'Common Humanity' subscale mean total scores against age

I am worried why I don't cry... and show more emotion, when a patient dies. Am I becoming desensitised already?

Frustration was a shared emotional experience of the work, particularly dealing with difficult and demanding patients and, in relation to a feeling of resentment and injustice towards colleagues who, they believed, did not work as hard as they did.
Getting frustrated with the work-load and, consequently, not being able to give care to the high standard that they wished to deliver.

I get quite upset that some patients are taking away so much time when you know that you've got things to do for other people. I find that quite frustrating

I'm down the other end of the ward struggling...drowning.

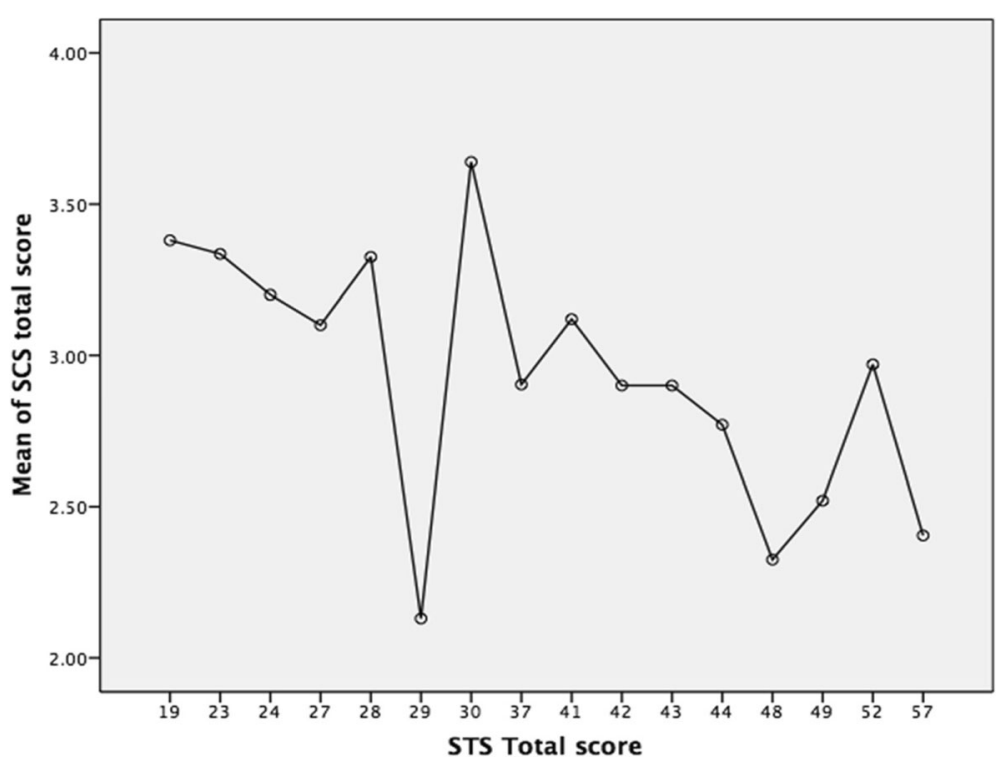

Fig. 12 Mean total scores of STSS and SCS 


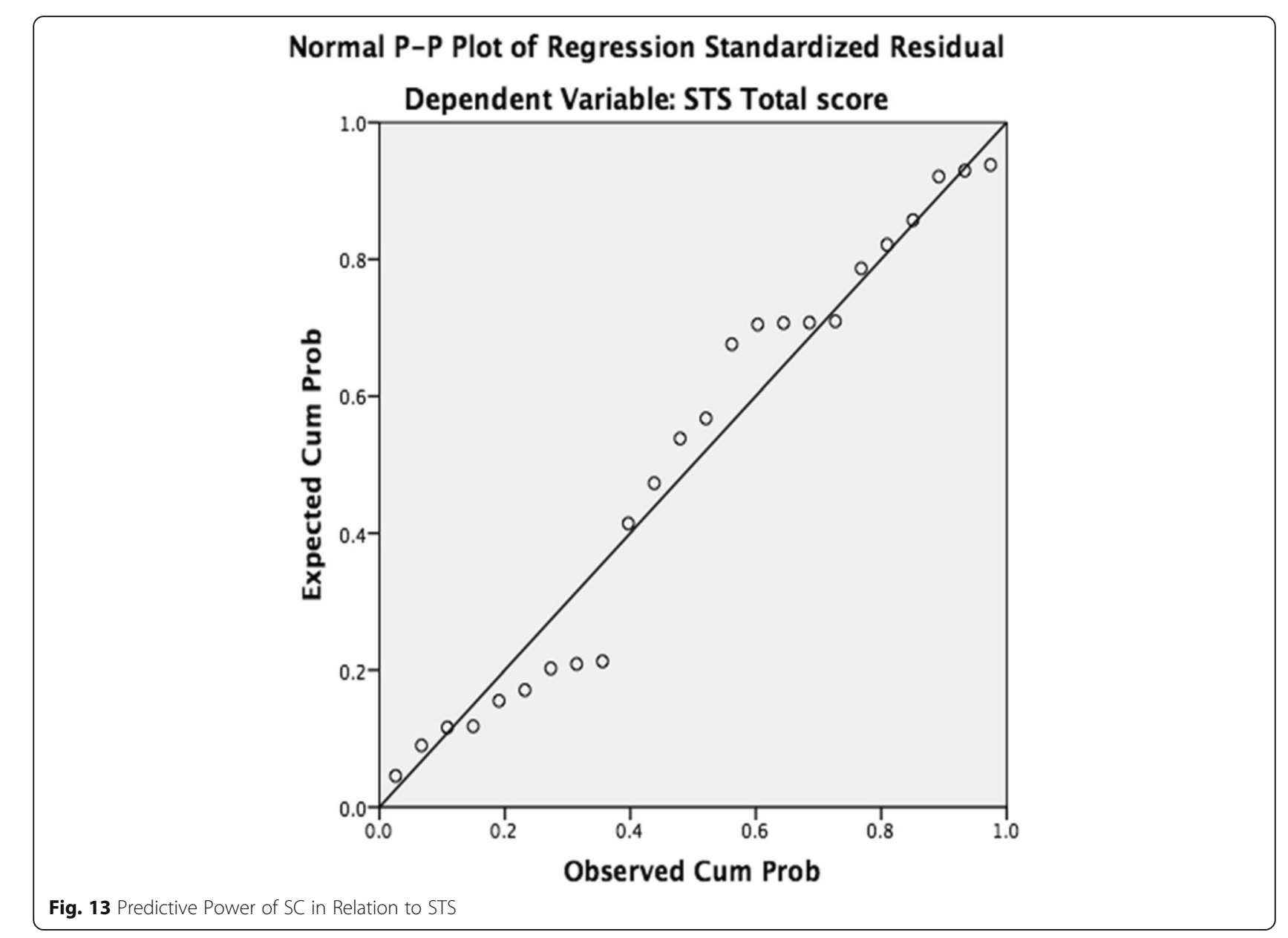

Expressions of low mood and unhappiness were felt, and feelings of heightened arousal and anticipatory anxiety were shown.

\section{Unhappy [with their job].}

So, the combination makes me not want to turn up for work, because you know already that you are going to walk into something that is going to be completely out of control.

Like that first day back at school.

\section{Cognitive effects}

All participants acknowledged that their thoughts and thinking styles were linked to their emotional feelings at work. These included intrusive thoughts and, being self-critical and judgmental towards themselves, thinking that they had not done their best at work and that they could have done more.
I've experienced intrusive thoughts about work and not having completed nursing tasks, which has fed into my dreams.

Interviewees believed that this detrimentally impacted on their home life on an evening when they should be switching off. The next day not feeling refreshed to take on what is thrown at me.

\section{I get critical and worried that I've missed something.}

I was lying there thinking, is it my fault, could I have done any more? But I did all that I could, so why was it not enough?

\section{Behavioural effects}

Related to working as a nurse in acute hospital medicine, the behaviours linked to the work-related emotional feelings and cognitions of the interviewees, were consciously 
self-protective and were largely avoidant or checking behaviours.

Feeling just really numb to it all. I've been avoidant of difficult patients (emotional avoidance).

[felt] panicked that I had not done something for a patient. So, I rang the ward in the middle of the nigh for peace of mind ('exaggerated checking behaviour').

\section{Physical effects}

The interviewees described how physically tired they were, not helped by having difficulties sleeping, and how the architecture of the hospital had had a physical impact.

I feel that my physical health has got worse since I started working there.

There are foot issues. Loads of staff tell me that they have foot and movement issues.

\section{Theme 3: factors contributing to the development of compassion fatigue}

During the interviews, the researcher asked participants to consider the underlying reasons for the disturbing and distressing effects of their nursing work, that they had described. Thematic analysis of their answers revealed six significant causative subthemes:
i. Self-Factors
ii. Ward Management Factors
iii. Hospital Organisational Factors
iv. Patient Factors
v. Environmental Factors
vi. External Factors

\section{Self-factors}

The interviewees revealed several factors related to their own attitudes and beliefs about nursing care, discussing that their very high standards of how I want the care to be were in conflict with their actual ability to meet these ideals.

Some set nursing care priorities to try and overcome this, but this strategy did not fit well with a desire to give high quality patient care.

It doesn't make me happy. I feel especially for those people who don't get enough of my care or don't get enough nurse time.

It was expressed that the ' $6 \mathrm{C}$ 's' (Nursing Midwifery Council, 2013), which had been drummed into them from day one, were based on unrealistic high expectations in relation to the provision of nursing care. It was felt that they did not support 'genuine and authentic care'.

Stress was also reported as having an impact on the nurses' ability to provide high standards of patient care.

When I am stressed I cannot guarantee that every last of the twelve patients [on my case list] gets, you know, more than their basic needs me. If I had six patient there would be a lot more connection and a lot more of me knowing what's going on.

\section{Ward management factors}

Weak management of staff was identified as contributing to stress during their nursing work and affecting their experience of Compassion Fatigue. Interviewees were particularly vocal about the reluctance of nurse managers and senior medical colleagues to deal effectively with difficult, disruptive patient behavior. They believed that reports by RGNs were being ignored, even when voiced through the policies and procedures set out by the Hospital Trust.

There is incident reporting, but it is rare that you see a response back from that. It just makes you feel undervalued and just that you have to put up with anything that is thrown at you and that the policy is not followed.

A colleague and I got attacked. Like this person was afterwards admitted a couple more times and I said please not to have him on the ward. And nothing happened.

I get quite upset that they [patients] are taking away so much time, when you know that you've got things to do for other people. I find that quite frustrating.

\section{Nothing happened...nobody [managers] does anything.}

This lack of managerial support caused the nurses anxiety.

And I find these things quite sad, that you can't, you know, there is not so much of a backing up for us when we say a certain patient is not, you know, conducive to the whole ward.

It was also disclosed that an unsatisfactory level of care provided to patients by some members of staff, particularly those employed from external Nursing Agencies, had not been addressed by staff management. 
I feel that some of the people who have been in the job longer, just don't care that much anymore. They've stopped having that, not all of them, but that feeling of being, I don't know, whether duty bound is the right word.

In my head, I am thinking that well they haven't come in on time, it is persistent, or they are not carrying out their care and then I have a look round and see how hard working some other colleagues are and how tired I feel. And I think, well, if you were doing your job effectively we wouldn't feel this way.

The thing is, when you speak to them [Agency staff], a lot of them do not care. It is sole destroying because they are basically doing a student nurse's job, not even that, and they are getting paid triple what we are.

Interviewees felt like they had been taken advantage of due to poor management and that this affected their mood and morale.

I did not leave. I could have been '7.30 I'm off' but I stayed to midnight because I was terrified of the thought that my patient was being left.

A glum feeling on the ward... a lot of people are worried about how it is going to be. Morale is just low. There's just this feeling.

Debriefing during a shift was seen as useful if stress levels were high, but finding an opportunity to do this was difficult, which impacted detrimentally on the newly qualified nurses.

We've got a couple of new girls and some of them literally break apart, so it's hard. I can empathise with how they feel.

Also voiced was a sense of feeling 'demoralised' when their efforts were not recognised and they had been trying so hard.

I was under the illusion that I had done the right thing and I had done a good job and then somebody came and really laid into me.

I would say most people on my ward do not really feel valued. And that is really hard because that could cause compassion fatigue in itself because you start to think "What am I doing this for?"

\section{Hospital organisational factors}

It was felt that the infamous madness of the general acute medical care environment, where patients have multiple, complex needs, along with staff shortages, low patient/staff ratios and, poor nurse skill mix and staff retention issues, detrimentally impacted upon the workload situation.

We are always down on staff. And now three of us are leaving, including me.

When it is just me and another health care assistant and we have got twelve patients to look for and seven of them need both of us to give them just basic care, such as hygiene needs like their bowels. You just can't do it. I wish I had six patients, then there would be a lot more connection and a lot more of me knowing what's going on.

On the books we are fully staffed, so if something happens it's not us.

The interviewees reported that they believed their work was target and protocol driven in order to protect the NHS and Trust from litigation, which was dehumanising and hindered individualised patient care.

You are like a robot. You are doing things because the guideline tells you to. It's so hard for me to do things out of clinical judgment, that would still be safe.

The feelings of being undervalued by their Trust employer was not helped by the hospital's award system - a system that nurses are rated as most caring by their patients and then celebrated by the Trust. Instead the participants saw it as unfair due to it being subjective and not reflecting the 'true winners'.

The problem with it is that nurses who really do the hard work, who really try their hardest to juggle all that need doing, might not always have the time to show that in depth relationship with the patients. So, the patient will not mention you. But, if you are the kind of person who chats a lot with patients and doesn't get much work done, they will like you because you are around.

Let's face it, the patients we have to care for the most are not those who are going to write letters to the Trust, because they are massively too ill to do anything about it themselves. 


\section{Patient factors}

Concern was raised by the interviewees that some patients greatly reduced a nurse's ability to establish a close, compassionate, caring relationship with that patient, due to the patient's negative attitude and behaviour towards their illness and healthcare. Furthermore, it was reported that threatening and inappropriate patient behaviour towards the nurses had a negative impact on the nurses.

They constantly call you in and are just rude and negative all the time. But of course, you have this duty of care to go in and see what people want.

If you've got particularly needy patients, or rude patients and/or relatives for that matter, I can come in and look at the board and it sets your day up from a bad start.

I was sitting in the office and one of the girls came in and she said, "Oh no, I can't be down in that section again, I really can't". She had been down with me in that section and I could see it in her. She's normally full of beans, but she had nothing to give that day. She needed to be moved somewhere else.

It's stressful when the odd patient sees me as their servant and even sometimes inappropriately touches [us], or says rude things [to us].

Interviewees also shared examples of how patient's expectations had become unrealistic.

I felt completely taken for granted.

[I felt] under-valued by patients, [even though I had] been running around like a blue-arsed fly all day.... and not had a break this morning

\section{Environmental factors}

Concerns and frustrations with their physical working environment, particularly the curved architectural shape of the ward, were highlighted.

It is just not practical, so that's frustrating. Not being able to see [your] patients from one end of the room to the other.

It is massively dangerous because you can't see what happens elsewhere. Can only ever see maybe six to nine feet ahead because of the curve.
I think in the last year loads of people have it [foot problems] and the Trust doesn't really recognise it. They must at some point acknowledge it as loads of staff tell me that they have foot and movement issues.

It was also expressed that the positioning of the staff room behind the ward's central nurses' station, means that staff are exposed and their protected break time can be interrupted by various other health care professionals.

\section{External factors}

Interviewees felt scrutinised on the patient care they gave because, given the history of complaints and published enquiries, the media had given the public a biased impression.

The media is toxic because it is feeding into people's minds the completely wrong impressions of what we try to do. [Patients] who have not been in hospital before and their loved ones look for mistakes and are not happy with things. Whereas the patients who are frequent visitors to the ward, they recognise that [we] are doing everything [we] can.

This makes a nurse hyper alert and conscious of what they have said and done. Consequently, they end up delivering care that is not authentic and not from the heart.

Research question 2: is self-compassion a coping strategy that enables acute medical care hospital nurses to mitigate the prevalence and levels of compassion fatigue?

Three dominant themes emerged from the coded data related to Self-Compassion (SC). These were:

- Recognition of the existence of Self-Compassion

- Is Self-Compassion relevant to nursing care?

- Achieving Self-Compassion.

\section{Theme 4: the existence of self-compassion}

The interviewees were not able to give an accurate definition of the concept of Self-Compassion, (SC).

I'm not totally sure.

So, liking yourself.

Theme 5: is self-compassion relevant to nursing care? As a consequence of the interviewees' lack of knowledge about Self Compassion, the research interviewer discussed the concept with them individually and they raised the following points. 
You have to look after yourself to be able to look after others effectively.

Compassionate towards yourself and feelings of positivity towards yourself. Because in the nursing profession it is so easy to grind yourself to the floor beat yourself up.

Accepting the multiple roles life brings. You can't be $100 \%$ in all of them.

Nurses are not very good at doing Self-Compassion.

\section{Theme 6: achieving self-compassion}

The researcher asked the interviewees to consider their personal approaches, as acute medical care nurses, to boosting their level of Self-Compassion, irrespective as to whether they were successful or not. Thematic analysis of their answers revealed four significant subthemes:

i. Self: Helpful Thinking Style ON Duty

ii. Self: Helpful Behaviours OFF Duty

iii. Self: Helpful Behaviours OFF Duty

iv. Helpful Managerial Behaviours

\section{Self: Helpful thinking style ON duty}

It was acknowledged by the interviewees that thinking realistically about the quality of nursing care delivered and being less self-critical but more self-forgiving, were useful coping strategies for the role of a nurse on an acute medical ward. The acceptance of putting things into perspective had come with time and experience.

I used to beat myself up a lot. But I think the more exposure I have to life as a nurse, I suppose, you realise that you just can't control everything. You just have to accept sometimes that you did everything you could.

Just realise that there are limitations in you as an individual and day to day life.

The importance of self-kindness through self-comforting and the benefit from 'Common Humanity' knowing that other people are feeling the same, that it happens to other people, that it is quite normal.

Self-briefing and self-reflection were seen as coping strategies that were helpful with the process of rationalising and putting feelings and emotions into perspective.
I've had a couple of times when I felt that this is really hard for me to do. But I've thought about it and why it was difficult for me to do and that helped.

\section{Self: Helpful Behaviours ON duty}

Using the support of other members of staff at all levels, particularly those who are experienced, to share thoughts, emotions and concerns about work, was viewed as very helpful, as well as organisational skills to control stress levels.

It's cathartic. It helped by talking to colleagues.

Best not to bottle things up. Knowing that other people are feeling the same and that they are all as scared as I am. You are not alone.

By trying to prioritise nursing tasks.

\section{Self: Helpful Behaviours OFF duty}

The interviewees understood the importance of trying to achieve a work-life balance by spending time to rest from work and getting out and about and not just sitting there thinking, wallowing.

A number of ways of achieving a work-life balance were discussed including days out and Yoga, to working less extra shifts when stressed and struggling emotionally and annual leave.

\section{Helpful managerial Behaviours}

Interviewees emphasised the importance of regular feedback and debriefing with senior staff, particularly for the younger ones and new ones coming in as it was helpful to put it all into perspective and realise that you are not alone.

It was suggested by the interviewees that having a protected break to shield them from various other health care professionals interrupting their time to take a breather which is not always easy.

The interviewees recommended that managers could demonstrate that they care and value their nursing staff by listening and acting on the acute care nurses' concerns and well-being. Suggestions were made as to how this could be achieved.

That it wasn't the same people always responsible for looking after that person (in relation to addressing the issue of demanding and abusive patients).

The importance of Compassion Fatigue being recognised more by their employer and of the need to improve the nursing staff"s awareness of its potential effect on 
them, through education and as part of the Preceptorship Programme and induction.

The issue there is who would recognise the importance of Self-Compassion and who would sit them down? I'm sure that would be really helpful.

The interviewees believed in the need for a mutually supportive and effective nursing team in order to limit Compassion Fatigue and encourage Self-Compassion.

\section{If say I've got two really good auxiliary nurses who are positive and really, really help me out and get me through the day, which they do, I automatically feel better.}

\section{Discussion}

\section{Introduction to discussion}

The purpose of this study was to investigate the environmental and psychosocial factors affecting the prevalence and levels of Compassion Fatigue (CF) in acute medical care nurses and to explore Self Compassion (SC) as a mitigating factor. The results indicated that, although the total acute medical care hospital nursing sample had CF levels of upper end of mild (STSS score of 37.54), the scores of nearly half these nurses indicated a moderate to very severe level of CF. Significantly, 37.5\% of the nurses' met all three criteria for a diagnosis of Post-Traumatic Stress Disorder (PTSD). Similar results were observed in a study by Beck, LoGuidice and Gable [84], which found a mean CF level in their nursing participants at the upper end of mild, yet 36\% met PTSD diagnostic criteria.

With regards to SC, this sample had levels at the lower end of the moderate range (2.87), lower than found by Heffernan et al. [60] (3.49, moderate SC), with $71 \%$ of nurses scoring 2.5 or above, indicating moderate to high levels of SC. However, some sub group scores suggested otherwise.

The results also suggest that six significant factors underlie the development and level of CF in acute medical care hospital nurses and that importantly, SC is a significant unique contributor to the prediction of CF.

\section{Discussion of results in relation to compassion fatigue}

Today's NHS nursing workforce is more varied in age than ever before, being made up of four different generations, each with different expectations, values, motivations and unique needs [85]. It is relevant that the results of this study suggest that age and years of experience working on an acute care ward, have a significant impact on a nurse's CF level.

Indeed, a positive correlation was found between CF and both age and years working in acute care medicine.
Consistent with earlier studies [86-90], this study's results indicate higher levels of CF in younger nurses with fewer years nursing experience. Nurses very new to the acute care setting had developed high levels of CF, in all likelihood due to their inexperience and the challenges brought by a busy ward environment [91]. A further rise in CF levels between the age of 26 and 30 coincided with the end of a supportive period of Preceptorship. On reaching 30 years, a drop in CF was noted, which may suggest adaptation to the demands of the job and the development of personal coping strategies, as illustrated by the interview data. However, as there was only one participant within this age range, it is not possible to generalise.

On reaching their 40s and increased work experience, the CF levels of the nurse participants rose sharply, which is not consistent with previous findings [86-90]. This may be due to these nurses' circumstances changing, with emotional, physical and financial stress [92]. They are required to meet new challenges in their lives: increased pressures from changes in family dynamics [93]; health problems and reduced stamina associated with the ageing process [94-96]; the pace of changes in new technology in the workplace [95]; age discrimination and managers who fail to value their experience [94, 95].

The study also reveals a relationship between shift patterns and $\mathrm{CF}$, with a moderately positive correlation $(r=0.436)$ between higher levels of CF and nurses working both short part-time shifts and those working full-time. However, those working between $22.5 \mathrm{~h}$ and $30 \mathrm{~h}$ per week show much lower levels of CF (no statistical correlation is found between these factors and $\mathrm{CF}$ ). A study by Yoder [89] found nurses working 8-h shifts had higher CF than those working 12-h shifts. Together these results may suggest that nurses working shorter hours may be doing so to fit in with the demands of family life and the attendant emotional pressures.

Interesting relationships between the individual symptoms of CF (Avoidance, Arousal and Intrusion) and the demographic and work-related characteristics of acute care nurses were found that, thus far, have not been found in previously published literature. Four significant, inter-related variables were found to affect the nurses' working lives and the prevalence and level of CF; Emotional Effects, Cognitive Effects, Behavioural Effects and Physical Effects.

Emotionally, nearly $60 \%$ of the nurses reported symptoms of heightened arousal - difficulty sleeping, irritability and hyper-vigilance being most frequently reported. Those aged 36 to 55 years of age experienced higher levels of Arousal, with RGN nurses having the highest levels. The interviews supported these findings, revealing difficulties in managing emotional feelings and heightened anticipation of stressful work situations. Emotional avoidance was also reported, with $46 \%$ of total nurse 
sample reporting a foreshortened future and emotional numbing by $38 \%$ of the sample. Interviewees commented that they were becoming desensitised and unmoved by the death of a patient.

In relation to Cognitive Effects, intrusive thoughts were reported by $70.8 \%$ of the nurse sample, with intrusive thoughts about clients the most frequently reported event. A statistically significant finding that nurses with a Nursing Degree are more susceptible to intrusive thoughts was supported by the interview data. It revealed that nurses recognise that their negative and self-critical thoughts and thinking styles are linked to their emotional feelings at work, with a detrimental impact on home life. This is reinforced by previous studies $[97,98]$. In particular, the interviewees emphasised emotive thoughts about frustrating interruptions to their ability to provide a very high level of care.

Behavioural avoidance of difficult patients was reported by nearly $40 \%$ of the nurses, with a marked increase in levels of avoidance between the age of mid-30s and mid-40s and particularly high levels for those aged 4145 years. This finding may be a reflection of a point in the nurses lives where they are having to navigate the stresses of new challenges, both in family dynamics and work.

The interviews revealed that work-related emotional feelings and cognitions often triggered self-protective behaviours, including checking their shift work by ringing the ward after reaching home.

In relation to Physical Effects, qualitative enquiry found that nurses suffer from chronic tiredness, disturbed sleep patterns caused by nightmares and raised heart rate. Consequently, they are not refreshed to embark on the challenges of the next working day. Further physical impacts are as a result of the architectural design of the hospital wards, with numerous foot issues being reported and concerns ignored.

The study also revealed factors that contributed to the development of CF in the acute care hospital nurses. These included Self-Factors, Ward Management Factors, Hospital Organisational Factors, Patient Factors, Environmental Factors and External Factors.

In relation to Self-Factors, the interview data revealed that the nurses found themselves unable to care for patients in a way that met their aspirations of delivering compassionate care, a dilemma that can lead to feelings of inadequacy, a contention supported by Smith et al., [99]. This was expressed by the interviewees who felt that their nursing care standards were compromised because of factors outside their control, including the stressful pressure of meeting the unrealistically high expectations of the 6Cs [6].

Physical isolation created by the curved design of the ward was of concern to the interviewees, as it put them at risk of experiencing violent and aggressive encounters, undetected by other staff. They gave examples of weak managerial staff support for nurses trying to deal with disruptive, abusive patients and visitors, reporting a tendency for nurse managers and senior medical staff not to respond proactively to ward staff concerns, which caused frustration and distress and, possibly contributed to increased CF. Studies have shown that personal experiences of violence and aggression in the workplace can lead to serious emotional consequences for nurses [100-102]. Importantly, Edward et al. [103] identified that younger, less experienced nurses and those working in physical isolation, are at particular risk of violence in the workplace, with incidents going unreported because staff don't feel confident that they will be supported properly by their senior managers. It is also noteworthy, that a recent study [104] has shown that feeling unappreciated, undervalued and having a lack of control over their nursing activities, can cause more stress to a nurse than the actual demands of the work itself.

Much has been written about the detrimental effects on staff of working within a healthcare organisation that places undue pressure on staff meeting targets [105]. Crawford, et al. [106], identified that it can severely compromise nurses' efforts to deliver compassionate care. This represented a recurring theme in the study interviews, as the nurses described their struggles to provide any more than just basic care, in a work environment that they described as protocol and target driven. In their view, this was to protect the NHS and their Trust employer from litigation, which subsequently had a dehumanising effect and hindered individualised patient care.

Nurses are often exposed to verbal, physical and sexual abuse from their patients [107]. Exposure to such threatening patient and relative behaviour is a considerable occupational stressor for nurses and other health-care professionals [107]. Such situations can lead to nurses avoiding and minimising their contact with inappropriate patients, which may adversely affect the quality of care the nurses provide for them (Felton, 1998). Examples from the study interviews included being touched inappropriately, threatening patient behaviour and being treated like a servant. It is important that health care organisations offer their employees access to support and counseling, with annual training to manage and prevent challenging behaviours. Additionally, health care educators should ensure that they provide for student nurses the opportunity to develop their skills and knowledge to avoid and manage such situations.

Traditional Nightingale ward design has been largely replaced in modern hospitals by single and four bedded rooms. The curved architectural design of the hospital wards in this study has raised concerns that patients and nurses are not continuously visible to others.

In relation to the External Factors that have contributed to CF in the nurses, the study results showed that 
participants felt that the patient-care they delivered was under the scrutiny of the toxic media, which led them to feeling hyper alert and conscious of the care they gave, leading to what they believed was inauthentic care that was not heartfelt. Certainly, negative coverage by the media and a number of high profile reports, such as 'The Francis Report' [3], have created an atmosphere of blame, rather than one where the wider concerns, such as the complexities of the care environment, are explored [18].

\section{Discussion of results in relation to self-compassion}

Self-Compassion (SC), in its simplest terms, is compassion directed inward, relating to oneself as the object of concern and care at a time of personal suffering [52]. There is a growing body of research evidence that suggests that SC is associated with fewer psychological symptoms [108], such as mood and anxiety disorders [52, 56, 57, 109]. and associated with positive psychological characteristics, such as emotional intelligence [60], life satisfaction and well-being $[56,57]$. In addition, it has been mooted that $\mathrm{SC}$ could be helpful in the prevention of CF in health-care professionals and hence promote compassionate patient care [64]. Indeed, this study shows the existence of a moderate, negative correlation between the two variables of SC and CF, with high levels of SC associated with lower levels of CF.

Although no statistical significance was found in this study between SC and, demographic and work-related characteristics, it did enable the identification of those individual nurses most likely to score higher or lower levels of SC. In many cases, on analysis of the results, levels of SC were shown to relate to levels of CF and, thereby, to the same demographic and work-related characteristics. For example, those nurses between 31 and 35 years had high levels of SC and low levels of CF, whilst those 51-55 years had a very low level of SC and severe level of CF.

The study revealed, through thematic analysis, those personal approaches of acute medical care nurses, whether successful or not, used to enhance levels of SC and the barriers to achieving it. Three of these themes were related to self-help approaches and the fourth was associated with aspects of managerial behaviour that they believed helped to enhance levels of SC.

A study by Duarte, Pinto-Gouveia and Cruz [110] suggests that being caring, supportive and understanding towards oneself (Self-Kindness - a characteristic associated with Self-Compassion), has the potential to be protective against CF. The results of this study indicated that acute medical care hospital nurses acknowledge that helpful thinking styles on duty at work include thinking realistically and putting things in perspective. These skills, that they believe develop with time and experience, are useful coping strategies in becoming less self-critical and more self-forgiving. They recognised the importance of cutting themselves some slack and showing themselves self-kindness through self-comforting. However, unfortunately, the quantitative results of this study suggested that the nurses were not succeeding in using these coping strategies, with many having low levels of Self-Kindness (46\%) and Mindfulness (30\%). Also, a significant number had high levels of Self-Judgment and Over-Identification, both indicative of low SC.

Within the context of acute medical care hospital nursing, the interviewees disclosed that they are self-critical and strict with themselves, believing they could have done things better. These negative attitudes were compounded by very high standards of how they want to deliver care and feeling under pressure from the unrealistically high expectations of the NMC ('6C's'), their employer and their patients.

Perfectionism in its simplest terms, is the tendency of individuals to set themselves high expectations, critically evaluating their ability to achieve their expectations [111]. But maladaptive perfectionism, involves the negative and unhealthy pursuit of unrealistic expectations and high levels of critical self-evaluation [112]. Perfectionism has been linked to depression [113], anxiety and worry $[114,115]$ and Obsessive- Compulsive Disorder (OCD) $[116,117]$.

Some perfectionistic OCD characteristics were demonstrated by the reports of the interviewees having a tendency to intrusive thoughts about their work, leading to excessive checking behaviours. This in turn, made them anxious and worried that their work-life was impacting on their home-life and on their ability to re-charge their batteries before the next working day. A high level of perfectionism is associated with low levels of mindfulness [118] and a tendency to adopt ruminative and brooding thinking, which is a maladaptive coping strategy [119]. Individuals who over-identify and ruminate have a tendency to feel overwhelmed and carried away by their negative emotional thoughts and reactions [110]. In this study, nearly a third (30\%) of the acute care nurses had low SCS scores on the Mindfulness subscale.

Self-Compassion involves recognising that the human experience is not faultless and that we are all imperfect. When individuals are in touch with their Common Humanity, they are able to remember that feelings of inadequacy, frustration and disappointment are universal [52]. The interviewees described how they benefited from an understanding of Common Humanity and knowing that other people are feeling the same and that it happens to other people, that it is quite normal. They used self-briefing and self-reflection as coping strategies to help rationalise their feelings and get their emotions into perspective. Lautebach and Becher [120] relate 
self-reflection to self-caring. By using the tool of purposeful self-reflection, the acute care nurses in this study are gaining self-knowledge and insight [121].

Acute medical care nurses are most likely to care for patients with multiple, complex needs, a situation in this study that is adversely impacted upon by staff shortages, low patient/staff ratios, poor nurse skill mix and staff retention issues. As a result, they describe themselves as being overwhelmed and stressed by the relentless excessively heavy workload. The interviewees report that helpful behaviours whilst on duty, such as their own organisational skills, were useful in controlling their work-related stress levels. These included prioritizing tasks and making lists. Also, that avoidance coping strategies could be used, such as not looking at the off duty too far ahead helped them to manage their expectations and the anxieties about working a shift with poor staffing levels.

The interviewees also reported that they thought it important to allow people to talk about and make sense of their emotional and psychological responses to stressful situations in acute medicine nursing. They found that by sharing their thoughts and concerns about their work, the support of other members of staff at all levels was often helpful in managing stress levels. The process of debriefing, whether at handover, in a sharing circle or in the staff room, was viewed as a valuable behavioural coping strategy, enabling them to know that other people are feeling the same and that they are not alone (Common Humanity).

Nurses often describe experiencing an imbalance in their work-life balance (WLB), which can detrimentally impact their stress levels [122]. Indeed, an RCN survey [123] showed that nearly two-thirds of nursing participants were not happy with their WLB.

The interviewees in this study also recognised the importance of personal helpful behaviours off duty to try and achieve a WLB, by proactively organising things on their days off, such as meeting up with colleagues outside of the work environment, going out for a meal, days out and exercising (such as running, yoga and meditation). Nurses often describe experiencing an imbalance in their work-life balance (WLB), which can detrimentally impact their stress levels [122]. Indeed, an RCN survey [123] showed that nearly two-thirds of nursing participants were not happy with their WLB.

The nurse participants also saw the benefit of working part-time and having flexible choices around their working shift patterns and the positive effect annual leave has in giving them the opportunity to put things into perspective through self-reflection and self-debrief. As $\mathrm{Hu}$ et al., [124] has highlighted, today's health care workforce is more diverse in age than ever before, each generational cohort having differing needs and expectations.
Millennials place particular emphasis on obtaining a WLB, seeing work as a means to enjoy life, but life comes first [125]. They are not afraid to work hard but in return they want flexibility to balance their work and home life [126]. These sentiments are reflected in the comments made by the study interviewees, all being in the Millennial age bracket.

The final theme, helpful managerial behaviours, highlighted concerns about lack of support and response to the concerns of the acute medical care nurses, leaving them feeling isolated, neglected and undervalued. Indeed, nearly a third of nurse participants had high levels of Isolation, indicating low SC. Effective ward management and leadership is crucial in helping support caregivers find coping mechanisms to manage the demands of their job [127]. Indeed, levels of manager support have been found to predict, significantly and negatively, the level of CF in Emergency Department Nurses [86].

\section{Recommendations for practice}

Throughout the discussion of the study results, evidence has emerged that it is essential for health care organisations, such as the NHS, to provide a healthy, supportive and compassionate working environment for their acute medical care hospital nurses, in which the nurses' emotional well-being is fully supported [127].

Nursing staff need to be made aware of the causes, symptoms and reinforcing cognitions and behaviours of $\mathrm{CF}$, so that they can consciously address them. This should start from the early days of nursing education, rather than only teaching the prescriptive requirements of the 6 Cs [6] as a goal for compassionate care. From the results of this study, the $6 \mathrm{Cs}$ policy evidently inspires young nurses but also frustrates them, when so many factors are found to confound its achievement.

Through confidential staff surveys, sources of CF in specific care settings can be identified, enabling curative and preventative strategies of staff training and managerial support to be put in place. Embedded in any attempts by healthcare providers to address CF in their staff should be the recognition of the significant and unique findings of this study. With results revealing a relationship between SC and CF and the ability of SC to be predictive of CF levels, it suggests that the SCS level could be used as a screening tool to identify those nurses who are more predisposed and vulnerable to developing $\mathrm{CF}$ and, subsequently, target support programmes can be implemented. Strategies designed by health organsations to improve levels of SC and encourage a more self-compassionate work-force must be developed taking into account the age profile of the nurses they employ. This can be achieved with a better understanding and consideration of the diverse needs and expectations of their multi-generational workforce. 
As demonstrated in this study, for levels of SC to improve, nurse management needs to accept the importance of actively encouraging opportunities for nurses to regularly debrief with one another in a safe and secure environment, to hear others similar experiences and realise that they are not alone. These opinions are supported by [128], who advocate the value of giving staff permission to share their honest emotions and feelings, to buffer the stresses of their work. Health Care Organisations and their leaders should be made more aware of the strategies that can facilitate such opportunities, including Schwartz Rounds, which are shown to reduce feelings of isolation [129] and Restorative Supervision, which is proven to reduce stress by $59 \%$ [130]. This could lead to the implementation of targeted support programmes, such as the Mindfulness-Based Stress Reduction (MBSR) programme [131] to increase SC levels and subsequently, minimise and buffer the development of $\mathrm{CF}$.

Encouragement of a more self-compassionate workforce through protected and uninterrupted breaks should be established in order for staff to recharge and refuel, along with the provision of flexible shift patterns to better balance work and home life.

A particular and growing factor influencing nurse distress and vulnerability to $\mathrm{CF}$, expressed by the interviewees in this study, was the feeling of being undervalued and being taken for granted by patients who have unrealistic expectations of the nursing role. Health care providers and policy makers need to continue to understand, through research, the influences on how patient expectations are formed, what these expectations are and develop methods to manage them. This can improve patient satisfaction and outcome and decrease liability. In turn, this has the potential to lead to situations in which patient-nurse relationships improve [132].

For nurses to be given the best prospect of learning to manage and overcome CF and provide consistent, compassionate $24 / 7$ care, the whole care system needs to make it a priority, including those in senior positions. Health care managers need to adopt behaviours that give their staff the permission and opportunity to develop an appropriate level of SC, whilst implementing strategies to remove barriers to achieving it and providing a supportive approach to those nurses identified as being overtly perfectionist and self-critical. Acknowledgment and celebration of the extraordinary work that acute medical care nurses achieve must be exhibited through meaningful recognition and, the implementation of organisational and cultural approaches that actively demonstrate the value and appreciation placed on nursing staff.

\section{Limitations}

Limitations of this study include the low response rate to the recruitment of study participants and the subsequent small sample size, both in the quantitative and qualitative elements of the study. Subsequently, the generalisability of these findings needs to be approached with caution. However, the results do suggest that further study is warranted to investigate the work-related, demographic characteristics and personality traits of acute care nurses, that make them more likely or less likely to be Self Compassionate. A subsequent profile could be developed which Health Organisations, Management and individual acute care nurses could use to help identify certain individuals who are more vulnerable to low levels of SC and therefore higher levels of CF.

Also in terms of generalisability, some variables, such as those nurses aged 51-55 years, that displayed interesting results in relation to levels of $\mathrm{SC}$ and $\mathrm{CF}$, are reported by very few acute medical care hospital nurses. However, this study is new in the research arena of SC and CF in acute medical care hospital nurses in the United Kingdom. Therefore, all results should be noted with interest, even if generalisability cannot be upheld.

It could be argued that this sample of acute medical care hospital nurses, from one urban acute medical care hospital, may not adequately represent the population of acute medical care hospital nurses, as CF and SC experiences of non-participants may differ from those who did participate. This limits the generalisability of the study and a study involving a larger number of nurse participants from multiple international acute medical care hospital wards would be helpful. The poor response rate also detrimentally impacted upon the ability to decide whether data collection was sufficient and data redundancy had been reached. However, even with this low response rate, statistically significant findings have been unearthed.

There may also be selection bias in this sample, as those who completed the questionnaires and were interviewed, may have self-selected to do so because they felt they were experiencing higher levels of CF in the work environment. However, the poor study response rate could also be a reflection of potential participants not having the energy or inclination to take part in the study because they were suffering from some level of stress and CF.

A final limitation is that the prevalence and level of CF and $\mathrm{SC}$ were measured at a single point in time, asking the participant to reflect on their past week. It is possible that an acute medical care hospital nurse's assessment of their thoughts and perceptions change over time due to fluctuations in the factors that influence levels and prevalence $\mathrm{CF}$ and $\mathrm{SC}$, such as their work-related conditions. A longitudinal study design may therefore be useful in determining a true indication of CF and SC prevalence and levels within a profession that experiences many variations on a daily basis.

Despite these limitations, this study is an original piece of research, with significant findings, which previous 
research has not investigated and unearthed. These findings warrant attention from Policy Makers, Health Care Organisations, Nurse Leaders and acute medical care hospital nurses themselves, along with further exploration.

\section{Conclusion}

Due to the low participation rate of this study and the impact this has on generalisability, pertinent future topics for researchers to examine could include a larger study to explore the thematic and statistical issues raised in this pilot study. Such a study could examine the influence of demographic and work-related characteristics, and include the effect of personality styles, such as maladaptive perfectionism (OCD), on the prevalence and levels of SC and CF. The study could also further investigate the factors identified in this study that influence the acute medical care hospital nurses distress and vulnerability to CF, as well as their personal approaches, whether successful or not, used to enhance their levels of $\mathrm{SC}$ and the barriers to achieving this.

The findings of this study also indicate that further studies are warranted which examine whether $\mathrm{SC}$ has the potential to be used to manage and treat those suffering from STS (CF) and PTSD. Evidence from this study used to draw this conclusion include; that $37.5 \%$ of the acute medical care hospital nurses in this study met all three criteria for a diagnosis of PTSD; that participants displayed OCD characteristics in the form of excessive checking behaviours to manage their intrusive thoughts; that OCD is a disorder which has been conceptually related to PTSD [133]; and finally, that this study has proven that SC has the ability to predict and buffer STS (CF).

Although there is emerging evidence suggesting a relationship between nurses' SC and compassionate patient care, it is limited [134]. Therefore, it would be relevant to recommend that further lines of inquiry examine the influence of this relationship in order to progress the need to move compassionate care towards more authentic compassionate care.

Although the small sample size has limited the generalisability of the findings of this mixed methods study, it is important to recognise that it is a unique and first known study of Compassion Fatigue and Self-Compassion in acute medical care hospital nurses. It has been able to fill gaps in literature exposed in the Integrative Literature Review and, its findings are of direct relevance and importance to healthcare providers, such as the NHS.

Although the problems for nursing staff in the NHS are complex and multifaceted, it is becoming clear that nursing staff with Self-Compassion have a better chance of managing the stresses of their work and care environment. Indeed, this study's findings have exposed the prevalence of Compassion Fatigue in acute care medical hospital ward nurses and it's damaging effects. It has also confirmed that Self-Compassion can have a moderating effect on CF and an ability to be predictive of CF.

The design of care environments and, the processes and culture of patient care provision, needs to recognise the existence of CF in nursing staff, as well as understanding how it can develop and the strategies best suited to minimise its development. For nurses to be fully present, connected and compassionate with their patients, care environments need to encourage the self-compassion and well-being of their nursing staff, enhancing their ability to be resilient to CF.

It is crucial for health care organisations, such as the NHS and their leaders, to recognise that patient care is provided in the context of organisations, not in isolation. Indeed, compassion and high-quality patient care can only be improved once the whole care system and environment is addressed, rather than finding fault at the individual level. This study has demonstrated that threatening cultures, weak leadership, an emphasis on task rather than process and a lack of meaningful recognition from both patients and employer, are a breeding ground for CF.

Improving levels of Self-Compassion in nurses predominantly involves changes in managerial behaviour and attitude. The most influential people in this system need to actively demonstrate their commitment to the behaviours and values that support caring [19] with the same level of response, focus and energy that they would give if research evidence showed that patient safety was being compromised and could lead to cases of litigation.

Within compassionate environments, acute medical care hospital nurses will be given a better opportunity to provide the authentic compassionate patient care that is both expected of their caring profession, which they aspire to deliver and, that is both satisfying to themselves, to their work colleagues and to their patients.

\section{Abbreviations \\ CF: Compassion Fatigue; ILR: Integrative Literature Review; PTSD: Post-Traumatic Stress Disorder; SC: Self-Compassion; SCS: Self-Compassion Scale; STS: Secondary Traumatic Stress; STSD: Secondary Traumatic Stress Disorder; STSS: Secondary Traumatic Stress Scale; WLB: Work-life balance}

\section{Acknowledgments \\ The Author would like to thank the participants for sharing their thoughts and experiences and, all nurses and health care professionals who work tirelessly, often under difficult circumstances, to provide patients with uncompromised high-quality compassionate care.}

Funding

The study was self-funded by the author KU.

\section{Availability of data and materials}

The qualitative datasets generated and/or analysed during this study are not publicly available as the participants were not informed that the data would be shared verbatim, but as anonymised quotations. The tables of quantitative data generated from analysing the completed questionnaires are available from the corresponding author $\mathrm{KU}$, on reasonable request. 


\section{Authors' contributions}

$\mathrm{KU}$ led this entire piece of research, from its conception, through to the study design, data collection and analysis, interpretation of results and the manuscript write up. The author read and approved the final manuscript.

\section{Ethics approval and consent to participate}

Ethical approval for this study was gained from the Science, Technology, Engineering and Mathematics Ethical Review Committee at the University at which the author was a postgraduate student (Committee reference number - ERN_15-0297S) and from the R\&D Governance Office at the hospital the participants worked (Committee reference number - RRK 5388). Participants gave written consent to participate in the study and how they wished their qualitative data to be used. All data in the manuscript is anonymised.

\section{Consent for publication}

All data in the manuscript is anonymised. The participants consented to participating in the study and interviewees completed a consent form outlining how they wished their data to be used.

\section{Competing interests}

The author declares that she has no competing interests.

\section{Publisher's Note}

Springer Nature remains neutral with regard to jurisdictional claims in published maps and institutional affiliations.

Received: 30 April 2018 Accepted: 15 June 2018

Published online: 29 June 2018

\section{References}

1. Department of Health. Six lives progress report. London: Department of Health; 2010.

2. Care Quality Commission. Dignity and nutrition inspection Programme: National Overview. London: Care Quality Commission; 2011.

3. Francis R. Report of the mid Staffordshire NHS foundation trust public inquiry. London: The Stationery office; 2013.

4. NMC. The code: standards of conduct, performance and ethics for nurses and midwives. 2008. Available at: http://www.nmc-uk.org/Publications/ Standards/The-code/Introduction (Accessed 10 Dec 2014).

5. The King's Fund. Patient centred leadership: rediscovering the purpose, report from the King's fund leadership review. London: The King's Fund; 2013. (Available at: http://www.kingsfund.org.uk/sites/files/kf/field/field_ publication_file/patient-centred-leadership-rediscovering-our-purposemay13.pdf (Accessed 11 Dec 2014).

6. Department of Health. Transforming care: a National Response to winterbourne view hospital. Department of Health review: final report. London: Department of Health; 2012.

7. General Medical Council and Nursing Midwifery Council. Joint statement of professional values. 2012. Available at: http:/www.gmcuk.org/Professional_ values_joint_statement_August_2012.pdf_49744505.pdf (Accessed 5 Mar 2016).

8. Goodrich J, and Cornwell J. Seeing the person in the patient: the point of care review paper. 2008. Available at: https:/www.kingsfund.org.uk/sites/ files/kf/Seeing-the-person-in-the-patient-The-Point-of-Care-review-paperGoodrich-Cornwell-Kings-Fund-December-2008.pdf (Accessed 10 Dec 2014).

9. Graber DR, Johnson JA. Spirituality and healthcare organisations. J Healthc Manag. 2001;46:39-52.

10. Thompson D, Ciechanowski PS. Attaching a new understanding to the patient-physician relationship in family practice. Am Board Fam Med. 2003;16(3):219-26.

11. Rave N, Geyer M, Reider B. Radical systems change: innovative strategies to improve patient satisfaction. J Ambul Care Manage. 2003;26(20):159-74.

12. Stewart MA. Effective physician-patient communication and health outcomes: a review. Can Med Assoc J. 1995;152(9):1423-33.

13. Goodrich J, Cornwell J. Seeing the person in the patient: The Point of Care review paper. London: The King's Fund; 2008. https://www.kingsfund.org.uk/ sites/default/files/Seeing-the-person-in-the-patient-The-Point-of-Care-reviewpaper-Goodrich-Cornwell-Kings-Fund-December-2008.pdf.

14. Shanafelt TD, West CP, Sloan JA. Career fit and burnout among academic faculty. JAMA. 2009;169(10):990-5.

15. Wright RB, Edwards N, Dowdeswell B, and McKee M. Investing in hospitals of the future. European observatory on health systems and policies. 2009.
16. Aiken LH, Sermeus W, Van den Heede K, Sloane DM, Busse R, McKee M, Bruyneel L, Rafferty AM, Griffiths P, Moreno-Casbas MT, Tishelman C, Scott A Brzostek T, Kinnunen J, Schwendimann R, Heinen M, Zikos D, Stromseng Sjetne I, Smith HL, Kutney-Lee A. Patient safety, satisfaction, and quality of hospital care: cross sectional surveys of nurses and patients in 12 countries in Europe and the United States. Br Med J. 2012;344(7851):e1717.

17. Parliamentary and Health Service Ombudsman. Care and compassion. Report of the ombudsman on ten investigations into NHS care of older people. London: Office of Parliamentary and Health Service Ombudsman; 2011. https://www.ombudsman.org.uk/sites/default/files/2016-10/ Care\%20and\%20Compassion.pdf (Accessed 11 Dec 2014).

18. Marrin M. Fallen angels - the nightmare nurses protected by silence. 2009. Available at: www.timesonline.co.uk/tol/comment/columnists/minette_ marrin/article6814962.ece (Accessed 16 Dec 2015).

19. Cornwell J. Developing a culture of compassionate care. 2012. Available at: http://www.kingsfund.org.uk/blog/2012/10/developing-culture-compassionate-care (Accessed $9 \operatorname{Jan}$ 2016).

20. Bevan G, Hood C. Have targets improved performance in the English NHS? Br Med J. 2006:332(7538):419-22.

21. Berwick D. A promise to learn - a commitment to act: improving the safety of patients in England. London: Department of Health; 2013.

22. Van Der Cingel M. Compassion and professional care: exploring the domain. Nurs Philos. 2009;10(2):124-36.

23. Van Der Cingel M. Compassion in care: a qualitative study of older people with a chronic disease and nurses. Nurs Ethics. 2011;18(5):672-85.

24. Lewin RA. Compassion: the core value that animates psychotherapy. Northvale: Jason Aronson Inc; 1996.

25. Crawford P, Brown B, Kvangarsnes M, Gilbert P. The design of compassionate care. J Clin Nurs. 2014;23(23-24):3589-99.

26. Cole-King A, Gilbert P. Compassionate care: the theory and the reality. J Holist Health Care. 2011;8:29-37.

27. Goetz JL, Keltner D, Simon-Thomas E. Compassion: an evolutionary analysis and empirical review. Psychol Bull. 2010;136:351-74.

28. Curtis K. Learning the requirements of compassionate practice: student vulnerability and courage. Nurs Ethics. 2014;21:210-23.

29. Lee $M$, Laurenson $M$, Whitfield C. Can compassion be taught to lessen the effects of compassion fatigue? J Care Serv Manag. 2012;6(3):121-30.

30. Bramley L, Matiti M. How does it really feel to be in my shoes? Patients' experiences of compassion within nursing care and their perceptions of developing compassionate nurses. J Clin Nurs. 2014;23(19-20):2790-9.

31. Schantz ML. Compassion: a concept analysis. Nurs Forum. 2007;42(2):48-55.

32. Peters MA. Compassion: an investigation into the experience of nursing faculty. Int J Hum Caring. 2006;10:38-4.

33. Straughair C. Exploring compassion: implications for contemporary nursing. Part 1. Br J Nurs. 2012a;21(3):160-4.

34. Goodrich J. What makes a compassionate relationship between caregiver and patient? Findings from the 'anniversary' Schwartz rounds. J Compassionate Health Care. 2016;391:8.

35. Figley C. Compassion fatigue: coping with secondary traumatic stress disorder in those who treat the traumatized. New York: Brunner-Routledge; 1995.

36. Bride BE, Robinson MM, Yegidis B, Figley C. Development and validation of the secondary traumatic stress scale. Res Soc Work Pract. 2004;14(1):27-35.

37. Boyle DA. Countering compassion fatigue: a requisite nursing agenda. Online J Issues Nurs. 2011;16(1):Manuscript 2.

38. Dominquez-Gomez E, Rutledge DN. Prevalence of secondary traumatic stress among emergency nurses. J Emerg Nurs. 2009;35:199-204.

39. Hooper C, Craig J, Janvin DR, Wetsel MA, Reimels E. Compassion satisfaction, burnout and compassion fatigue among emergency nurses compared with nurses in other selected inpatient specialties. J Emerg Nurs. 2010;36(5):420-7.

40. Joinson C. Coping with compassion fatigue. Nursing. 1992;2(4):116-22.

41. Quinal L, Harford S, Rutledge DN. Secondary traumatic stress in oncology staff. Cancer Nurs. 2009;32(4):E1-7.

42. Aycock N, Boyle D. Interventions to manage compassion fatigue in oncology nursing. Clin J Oncol Nurs. 2009;13(2):183-91.

43. Pfifferling J-H, Gilley K. Overcoming compassion fatigue. Fam Pract Manag. 2000;7:39-44.

44. Schwam $\mathrm{K}$. The phenomenon of compassion fatique in perioperative nursing. AORN J. 1998;68(4):642-8.

45. Bride BE, Radey M, Figley CR. Measuring compassion fatigue. Clin Soc Work J. 2007;35(3):155-63. 
46. Burtson PL, Stichler JF. Nursing work envornment and nurse caring; relationship among motivational factors. J Adv Nurs. 2010;66(8):1819-31.

47. Potter P, Deshields T, Divanbeigi J, Berger J, Cipriano D, Norris L, Olsen S Compassion fatigue and burnout: prevalence among oncology nurses. Clin J Oncol Nurs. 2010;14(5):56-62.

48. Showalter SE. Compassion fatigue: what is it? Why does it matter? Recognising the symptoms, acknowledging the impact, developing the tools to prevent compassion fatigue and strengthen the professional already suffering from the effects. Am J Hosp Palliat Med. 2010;27(4):239-42.

49. Thomas RB, Wilson JP. Issues and controversies in the understanding of compassion fatigue, vicarious traumatization and secondary traumatic stress disorder. Int J Emerg Ment Health. 2004;6(2):81-92.

50. Bush NJ. Compassion fatigue: Are you at risk? Oncol Nurs Forum. 2009;36(1):24-8.

51. Sawbridge $Y$, Hewison A. Time to care? Responding to concerns about poor nursing care. Birmingham: HSMC Policy Paper 12, Health Services Management Centre, University of Birmingham; 2011. Available at: https:// www.birmingham.ac.uk/Documents/college-social-sciences/social-policy/ HSMC/publications/PolicyPapers/policy-paper-twelve-time-to-care.pdf.

52. Neff $K$. The development and validation of a scale to measure selfcompassion. Self Identity. 2003a;2:223-50.

53. Neff K. Self-compassion: an alternative conceptualisiton of a healthy attitude toward oneself. Self. 2003b;2:85-102.

54. Germer CK, Neff KD. Self-compassion in clinical practice. J Clin Psychol. 2013;69(8):1-12.

55. Leary MR, Tate EB, Adams C, Allen AB, Hancock J. Self- compassion and reactions to unpleasant self-relevant events: the implications of treating oneself kindly. J Pers Soc Psychol. 2007;92(5):887-904.

56. Neff KD, Rude SS, Kirkpatrick K. An examination of self-compassion in relation to positive psychological functioning and personality traits. J Res Pers. 2007a;41:908-16

57. Neff KD, Kirkpatrick K, Rude SS. Self-compassion and its link to adaptive psychological functioning. J Res Pers. 2007b;41:139-54.

58. Neff KD, Vonk R. Self-compassion versus global self-esteem: two different ways of relating to oneself. J Pers. 2009;77:23-50.

59. Gilbert P, Procter S. Compassionate mind training for people with high shame and self-criticism: overview and pilot study of a group therapy approach. Clin Psychol Psychother. 2006;13:353-79.

60. Heffernan M, Griffin MTQ, McNulty R, Fitzpatrick JJ. Self-compassion and emotional intelligence in nurses. Int J Nurs Pract. 2010;16:366-73.

61. Hollis-Walker L, Colosimo K. Mindfulness, self-compassion, and happiness in non-meditators: a theoretical and empirical examination. Personal Individ Differ. 2011;50(2):222-7.

62. Neff KD, and Beretvas SN. The role of self-compassion in romantic relationships. 2012

63. Neff KD, Pommier E. The relationship between self-com- passion and otherfocused concern among college undergraduates, community adults, and practicing meditators. Self Identity. 2012;12(2):160-76.

64. Gustin W, Wagner L. The butterfly effect of caring - clinical nursing teachers' understanding of self-compassion as a source to compassionate care. Scand J Caring Sci. 2013;27(1):175-83.

65. Chen H-C, Heaston S, Hunsaker S, Maughan D. Factors that influence the development of compassion fatigue, burnout and compassion satisfaction in emergency department nurses. J Nurs Scholarsh. 2014;47(2):186-94.

66. Karlsson M, Mayhew L, Plumb R, Rickayzen B. An international comparison of long-term care arrangements: an investigation into the equity, efficiency and sustainability of the long-term care systems in Germany, Japan, Sweden, the United Kingdom and the United States. London: Cass Business School; 2004. Available at: https://www.researchgate.net/publication/ 238788017_An_International_Comparison_of_Long-Term_Care_ Arrangements (Accessed 8 Feb 2016).

67. Adams CE, Leary MR. Promoting self-compassionate attitudes toward eating among restrictive and guilty eaters. J Soc Clin Psychol. 2007:26(10):1120-44.

68. McMullen L. Oncology nursing and compassion fatigue: caring until it hurts. Who is caring for the caregiver? Oncol Nurs Forum. 2007;34:491-2.

69. Morse JM. Principles of mixed methods and multimethod research design. In: Tashakkori A, Teddlie C, editors. Handbook of mixed methods in social \& behavioral research. Thousand Oaks: Sage; 2003. p. 189-208.

70. Beck C. Secondary traumatic stress in nurses: a systematic review. Arch Psychiatr Nurs. 2011;25(1):1-10

71. Broyles LM, Rodriguez KL, Price PA, Bayliss NK, Sevick MA. Overcoming barriers to the recruitment of nurses as participants in health care research. Qual Health Res. 2011;21:1705-18.
72. American Psychological Association. Diagnostic and statistical manual of mental disorders (DSM). 2000. Available at: http://dsm.psychiatryonline.org/ doi/book/10.1176/appi.books.9780890425596 (Accessed 17 Jun 2014).

73. Ting $L$, Jacobson JM, Sanders S, Bride B, Harrington D. The secondary traumatic stress scale (STSS): confirmatory factor analyses with a national sample of mental health social workers. J Hum Behav Soc Environ. 2005;11(3):177-94.

74. Stamm BH. Measuring compassion satisfaction as well as fatigue: developmental history of the compassion satisfaction and fatigue test. In: Figley C, editor. Treating compassion fatigue. New York: Brunner-Routledge; 2002. p. 107-22.

75. Badger K, Royse D, Craig C. Hospital social workers and indirect trauma exposure: an exploratory study of contributing factors. Health Soc Work. 2008:33(1):63-72.

76. Chilban J. Interviewing in depth: the Interactive Relational approach. Thousand Oaks: Sage; 1996.

77. Rubin HJ, Rubin IS. Qualitative interviewing - the art of hearing data. 2nd ed. Thousand Oaks, London, New York: Sage Publications; 2005.

78. Polit DF, Beck CT. Nursing research: generating and assessing evidence for nursing practice. 9th ed. Philadelphia: Wolsters Kluwer Health/Lippincott Williams and Wilkins; 2012

79. Clancy M. Is reflexivity the key to minimising problems of interpretation in phenomenological research? Nurs Res. 2013;20(6):12-6.

80. Cohen J. Statistical power analysis for the behavioral sciences. New York: Routledge Academic; 1988

81. Braun V, Clarke V. Using thematic analysis in psychology. Qual Res Psychol. 2006;3(2):77-101.

82. Bride BE, Robinson MM, Yegidis B, Figley CR. Development and validation of the secondary traumatic stress scale. Res Soc Work Pract. 2004;14:27-35.

83. Dibley L. Analyzing narrative data using McCormack's lenses. Nurs Res. 2011:18(3):13-9.

84. Beck CT, LoGiudice J, Gable RK. A mixed-methods study of secondary traumatic stress in certified nurse-midwives: shaken belief in the birth process. J Midwifery Womens Health. 2015;60(1):16-23.

85. Guthrie L. The next generation of workers. Escondido: Ken Blanchard Companies; 2009.

86. Hunsaker S, Chen H, Maughan D, Heaston S. Factors that influence the development of compassion fatigue, burnout, and compassion satisfaction in emergency department nurses. J Nurs Scholarsh. 2015;47:186-94.

87. Kelly R, Spencer. Predictors of compassion fatigue and compassion satisfaction in acute care nurses. J Nurs Scholarsh. 2015:47(6):522-8.

88. Sacco TL, Ciurzynski SM, Harvey ME, Ingersoll GL. Compassion satisfaction and compassion fatigue among critical care nurses. Crit Care Nurse. 2015:35(4):32-42.

89. Yoder EA. Compassion fatigue in nurses. Appl Nurs Res. 2010;23(4):191-7.

90. Von Rueden KT. Hinderer KA, McQuillan KA, Murray M, Kramer B, Gilmore R, Friedmans E. Burnout, compassion fatigue, compassion satisfaction, and secondary traumatic stress in trauma nurses. J Trauma Nurs. 2010;21(4):160-9.

91. Delaney C. Walking a fine line: graduate nurses' transition experiences during orientation. J Nurs Educ. 2003:42(10):437-43.

92. Pitsenberger DJ. Juggling work and elder caregiving: work-life balance for aging American workers. AAOHN J. 2006:54(4):181-5.

93. Leggett $W$. British social democracy beyond new labour: entrenching a progressive consensus. Br J Politics Int Relat. 2007;9(3):346-64.

94. Storey C, Cheater F, Ford J, Leese B. Retention of nurses in the primary and community care workforce after the age of 50 years: database analysis and literature review. J Adv Nurs. 2009;65:1596-160.

95. Wray J, Aspland J, Gibson H, Stimpson A, Watson R. "A wealth of knowledge": A survey of the employment experiences of older nurses and midwives in the NHS. Int J Nurs Stud. 2009;46:977-85.

96. Bennett J, Davey B, Harris R. Nurses working in mid-life. London: Nursing Research Unit, King's College London; 2007.

97. Nolen-Hoeksema S. The role of rumination in depressive disorders and mixed anxiety/depressive symptoms. J Abnorm Psychol. 2000;109(3):504-11.

98. Brosschot JF, Pieper S, Thayer JF. Expanding stress theory: prolonged activation and perseverative cognition. Psychoneuroendocrinology. 2005:30(10):1043-9.

99. Smith S, James A, Brogan A, Adamson E, Gentleman M. Reflections about experiences of compassionate care from award winning undergraduate nurses - what, so what.....Now what? J Compassionate Health. 2016;3:6.

100. Fisher JE. Fear and learning in mental health settings. Int J Ment Health Nurs. 2002;11:128-34. 
101. Reininghaus U, Craig T, Gournay K, Hopkinson P, Carson J. The high secure psychiatric hospitals' nursing staff stress survey 3: identifying stress resistance resources in the stress process of physical assault. Personal Individ Differ. 2007:42:397-408.

102. Kamchuchat C, Chongsuvivatwong V, Oncheunjit S, Yip TW, Sangthong R. Workplace violence directed at nursing staff at a general hospital in southern Thailand. J Occup Health. 2008;50:201-7.

103. Edward K, Ousey K, Warelow P, Lui S. Nursing and aggression in the workplace: a systematic review. Br J Nurs. 2014;23(12):653-9.

104. Johnston D, Bell C, Jones M, Farquharson B, Allan J, Schofield P, Ricketss I, Johnston M. Stressors, appraisal of stressors, experienced stress and cardiac response: a real-time, real-life investigation of work stress in nurses. Ann Behav Med. 2016;50(2):187-97.

105. Bevan G, Hood C. What's measured is what matters: targets and gaming in the English public health care system. Public Adm. 2006;84(3):517-38.

106. Crawford ER, LePine JA, Rich BL. Linking job demands and resources to employee engagement and burnout: a theoretical extension and meta-analytic test. J Appl Psychol. 2010;95(5):834-48.

107. Stevenson KN, Jack SM, O'Mara L, Le Gris J. Registered nurses' experiences of patient violence on acute care psychiatric inpatient units: an interpretive descriptive study. BMC Nurs. 2015;14(1):35.

108. MacBeth A, Gumley A. Exploring compassion: a meta-analysis of the association between self-compassion and psychopathology. Clin Psychol Rev. 2012;32(6):545-52

109. Raes F, Pommier E, Neff KD, Van Gucht D. Construction and factorial validation of a short form of the self-compassion scale. Clin Psychol Psychother. 2011;18:250-5.

110. Duarte J, Pinto-Gouveia J, Cruz B. Relationships between nurses' empathy, self-compassion and dimensions of professional quality of life: a cross-sectional study. Int J Nurs Stud. 2016;60:1-11.

111. Frost RO, Heimburg RG, Holt CS, Mattia Jl, Neubauer AL. A comparison of two measures of perfectionism. Personal Individ Differ. 1993;14:119-26.

112. Hamachek DE. Psychodynamics of normal and neurotic perfectionism. Psychology. 1978;15:27-33.

113. Frost RO, Marten P, Lahart C, Rosenblate R. The dimensions of perfectionism. Cogn Ther Res. 1990;14:449-68.

114. Juster HR, Heimberg RG, Frost RO, Holt CS, Mattia JI, Faccenda K. Social phobia and perfectionism. Personal Individ Differ. 1996;21:403-10.

115. Lundh LG, Ost LG. Recognition bias for critical faces in social phobics. Behav Res Ther. 1996;34:787-94.

116. Frost RO, Steketee G. Perfectionism in obsessive-compulsive disorder patients. Behav Res Ther. 1997;35(4):291-6.

117. Antony MM, Roth D, Swinson RP, Huta V, Devins GM. Illness intrusiveness in individuals with panic disorder, obsessive-compulsive disorder, or social phobia. J Nerv Ment Dis. 1998;186:311-5.

118. Perolini CM. Mindfulness and perfectionism as predictors of physical and psychological well-being in college students: ETD Collection for Fordham University; 2012. http://www.inquiriesjournal.com/articles/801/4/ mindfulness-and-perfectionism

119. Blankstein KR, Lumley CH. Multidimensional perfectionism and ruminative brooding in current dysphoria, anxiety, worry, and anger. J Ration Emot Cogn Behav Ther. 2008;26(3):168-93.

120. Lautebach SS, Becher PH. Caring for self: becoming a self-reflective nurse. Holist Nurs Pract. 1996;10(2):57-68.

121. Palmer PJ. The courage to teach: exploring the inner landscape of a teacher's life. San Francisco: Jossey-Bass; 2007.

122. Mullen K. Barriers to work-life balance for hospital nurses. Workplace Health Saf. 2015;63(3):96-9.

123. RCN (2013) RCN employment survey 2013. Available at https://www.rcn.org.uk/ professional-development/publications/pub-004503. (Accessed 9 Sept 2016).

124. $\mathrm{Hu} J$, Herrick C, Hodgin KA. Managing the multi- generational nursing team. Healthc Manag. 2004;23(4):334-40.

125. Hansen RS. Perception vs. reality: 10 truths about the generation $Y$ workforce. 2008. Available at: https://www.livecareer.com/career/advice/ jobs/gen-y-workforce. (Accessed 9 Sept 2016).

126. Lavoie-Tremblay M, Leclerc E, Marchionni C, Drevniok U. The needs and expectations of generation $Y$ nurses in the workplace. J Nurses Prof Dev. 2010;26(1):2-8

127. Maben J, Adams M, Peccei R, Murrells T, Robert G. Poppets and parcels': the links between staff experience of work and acutely ill older peoples' experience of hospital care. Int J Older People Nursing. 2012;7:83-94.
128. Grandey A, Groth M. From bad to worse: negative exchange spirals in employee-customer service interactions. Organ Psychol Rev. 2012;2(3):208-33.

129. Goodrich J. Supporting hospital staff to provide compassionate care: do Schwartz center rounds work in English hospitals? J R Soc Med. 2012;105(3):117-22.

130. Wallbank S, Preece E. Evaluation of clinical supervision given to health visitor and school nurse leadership participants. West Midlands: NHS and Worcester University, Birmingham; 2010.

131. Cohen-Katz J, Wiley SD, Capuano T, Baker DM, Kimmel S, Shapiro S. The effects of mindfulness-based stress reduction on nurse stress and burnout, part II: a quantitative and qualitative study. Holist Nurs Pract. 2005;19(1):26-35.

132. McKinley S, Nagy S, Stein-Parbury J, Bramwell M, Hudson J. Vulnerability and security in seriously ill patients in intensive care. Intensive Crit Care Nurs. 2002;18(1):27-36.

133. Dykshoom KL. Trauma-related obsessive-compulsive disorder: a review. Health Psychol Behav Med. 2014;2(1):517-28.

134. Mills J, Wand T, Fraser JA. On self-compassion and self-care in nursing: selfish or essential for compassionate care? Int J Nurs Stud. 2015;52(4):791-3.

\section{Ready to submit your research? Choose BMC and benefit from:}

- fast, convenient online submission

- thorough peer review by experienced researchers in your field

- rapid publication on acceptance

- support for research data, including large and complex data types

- gold Open Access which fosters wider collaboration and increased citations

- maximum visibility for your research: over $100 \mathrm{M}$ website views per year

At BMC, research is always in progress.

Learn more biomedcentral.com/submissions 